\title{
ArcheoSciences
}

Revue d'archéométrie

$36 \mid 2012$

Varia

\section{Présentation et application d'une méthodologie de caractérisation physico-mécanique de petits échantillons pour l'étude des matériaux du patrimoine bâti}

Presentation and application of a methodology of physical and mechanical characterization on small samples to study the materials of the built heritage

\section{Fabrice Dagrain}

\section{(2) OpenEdition}

Journals

Édition électronique

URL : https://journals.openedition.org/archeosciences/3865

DOI : 10.4000/archeosciences.3865

ISBN : 978-2-7535-2243-5

ISSN : 2104-3728

Éditeur

Presses universitaires de Rennes

Édition imprimée

Date de publication : 31 décembre 2012

Pagination : 191-208

ISBN : 978-2-7535-2241-1

ISSN : $1960-1360$

Référence électronique

Fabrice Dagrain, «Présentation et application d'une méthodologie de caractérisation physico-

mécanique de petits échantillons pour l'étude des matériaux du patrimoine bâti », ArcheoSciences [En ligne], 36 | 2012, mis en ligne le 31 décembre 2014, consulté le 28 janvier 2022. URL : http:// journals.openedition.org/archeosciences/3865; DOI : https://doi.org/10.4000/archeosciences.3865 


\title{
Présentation et application d'une méthodologie de caractérisation physico-mécanique de petits échantillons pour l'étude des matériaux du patrimoine bâti
}

\author{
Presentation and Application of a Methodology of Physical \\ and Mechanical Characterization on Small Samples to Study the Materials \\ of the Built Heritage
}

\author{
FabriceD AGRAIN*
}

\begin{abstract}
Résumé : La détermination des propriétés physico-mécaniques des matériaux de construction se fait classiquement par l'utilisation de techniques de caractérisation qui sont standardisées et qui nécessitent de préparer des échantillons de géométries et de tailles normalisées. Ce type d'essais ne peut malheureusement pas s'appliquer à des matériaux dont il n'est pas possible d'obtenir des échantillons en quantité et taille suffisante. C'est notamment le cas dans le domaine de la conservation et de la restauration du Patrimoine. Or, dans ce domaine particulier, la connaissance de l'état de conservation ou d'altération des matériaux est cruciale pour décider des traitements à appliquer afin de pérenniser les ouvrages historiques dont nous sommes les héritiers. De nombreux chercheurs ont dès lors testé et mis au point un certain nombre de méthodes permettant de caractériser, de manières alternatives, les matériaux de construction sur la base d'échantillons de très petite taille. Une méthodologie a été mise au point dans le Département de Génie Civil et Mécanique des Structures de l'Université de Mons. Quelques cas d'applications pratiques sur des matériaux historiques sont traités notamment pour illustrer les potentialités de la méthodologie à fournir un certain nombre d'informations quant aux matériaux que nous retrouvons dans le patrimoine bâti, ou qui peuvent être mis à jour lors de fouilles archéologiques. L’utilisation de cette méthodologie devrait permettre de compléter les études archéologiques de sites historiques en fournissant des informations utiles aux archéologues, aux architectes et autres entrepreneurs et ingénieurs qui devront faire en sorte de conserver et pérenniser notre patrimoine historique.

Abstract: The determination of the physico-mechanical properties of building materials is usually done by the use of characterization techniques which are standardized and which require to prepare samples of standardized geometries and sizes. This type of tests cannot unfortunately be applied to materials of which it is not possible to obtain samples in sufficient quantity and size. It is particularly the case in the field of the conservation and the restoration of the Inheritance. However, in this particular field, the knowledge of the state of conservation or deterioration of materials is crucial to decide treatments to apply in order to perennialize the historical works. Many researchers have consequently tested and developed number of methods to characterize, in alternative ways, the building materials on the basis of sample of very small size. A methodology has been developed in the Department of Civil Engineering and Structural Mechanics of the University of Mons. Some case studies on historical materials are treated to illustrate the potentialities of the methodology to assess information on materials which can be found in the built inheritance, or which can be discovered during archaeological excavations. The use of this methodology should make it possible to supplement the archaeological studies of historic sites by providing useful information to the archaeologists, to the architects and to other contractors and engineers who will have to preserve and perennialize our historical heritage.
\end{abstract}

Mots clé : caractérisation physico-mécanique, matériaux, patrimoine, petits échantillons, conservation, restauration.

Keywords: physico-mechanical characterization, materials, inheritance, small samples, conservation, restoration.

* Chercheur Qualifié, Département de Génie Civil et Mécanique des Structures, Université de Mons, Faculté Polytechnique - rue du Joncquois, 53, 7000 Mons,B elgique.( fabrice.dagrain@umons.ac.be) 


\section{INTRODUCTION}

La caractérisation physique et mécanique des matériaux naturels ou reconstitués est un moyen utile pour comprendre pourquoi des matériaux anciens sont parvenus jusqu'à nos jours, et ce malgré les dommages qui peuvent leur être provoqués par différents types de dégradations physico-chimiques ou mécaniques. Les méthodes de caractérisation sont nombreuses et généralement normalisées. Les normes varient fortement en fonction du type de matériaux à étudier, de leurs d'applications, ou encore tout simplement du type de propriétés à déterminer. Elles dépendent encore du domaine d'application et même des pays dans lesquels elles sont rédigées ou appliquées. Les normes sont très strictes tant avec les conditions expérimentales à respecter, qu'avec le nombre d'échantillons à tester ou la forme et la taille qu'ils doivent respecter.

Dans le domaine de l'archéométrie, les matériaux qui peuvent être prélevés, ou qui sont disponibles pour les études des chercheurs sont généralement en nombre limité, mais aussi de taille réduite. Il est ainsi très rare de pouvoir réaliser des caractérisations physico-mécaniques sur des matériaux anciens retrouvés dans des fouilles ou sur des ouvrages en restauration. Pourtant, les technologies actuelles permettent de caractériser physiquement et mécaniquement des échantillons de taille relativement réduite. Le Département de Génie Civil et Mécanique des Structures de l'université de Mons a mis au point une méthodologie qui lui est propre et qui permet d'accéder à diverses propriétés physicomécaniques de matériaux naturels ou reconstitués sur base d'échantillons de taille très réduite.

\section{DesCription de la méthodologie}

La méthodologie en question a été mise au point dans le cadre d'une recherche postdoctorale visant l'utilisation d'une technique de grattage pour caractériser les propriétés des matériaux de construction (Projet First Post Doc GEOMAT $^{1}$ ) et mise en application dans le cadre d'une recherche visant une meilleure compréhension des matériaux dans les structures anciennes afin de garantir la compatibilité entre matériaux anciens et matériaux de restauration

1. Projet First Post Doc Geomat - Caractérisation mécanique non-conventionnelle des géomatériaux à l'aide d'un test de grattage. Décembre 2007 novembre 2009. Les procédures expérimentales développées dans le cadre de ce projet ont permis de mettre au point une démarche scientifique permettant de caractériser des échantillons de petites tailles qui ne peuvent pas être testés classiquement par des méthodes normalisées.
(Projet COMAREST2 ${ }^{2}$. La méthodologie permet d'accéder à la fois à des propriétés physiques telles que la densité, la porosité, la capillarité, la dureté ou encore la vitesse sonique, et à des propriétés mécaniques dont l'énergie spécifique intrinsèque, l'angle de frottement interne et la résistance au forage. L'ensemble et l'ordre des procédures expérimentales permet en outre de réaliser l'ensemble des mesures sur le même échantillon.

Nous synthétisons ci-après les travaux réalisés dans le cadre de ces études, et pour plus de détails, nous renverrons les lecteurs aux références mentionnées dans le texte.

\section{Taille et géométrie des échantillons}

La taille et la géométrie des échantillons pouvant être analysés avec la méthodologie mise au point ne sont pas limitatives du point de vue de la réalisation des tests. De

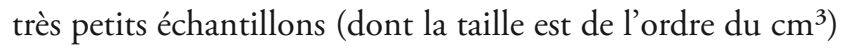
peuvent aussi bien être testés que de plus grands $\left(\mathrm{dm}^{3}\right.$ ou plus). Les échantillons peuvent être prélevés manuellement (fig. 1) ou par carottage (fig. 2).

Certains tests de la méthodologie nécessitent cependant des échantillons de taille minimale (pour le test sonique par exemple) ou de géométrie définie (notamment pour le test de remontée capillaire).

Travailler sur de petits échantillons apporte néanmoins un lot d'inconvénients dont il faut s'accommoder. Une attention toute particulière doit être apportée à la représentativité des échantillons sélectionnés par rapport à l'ensemble structurel devant être caractérisé. Plus l'échantillon à étudier est petit, plus le risque de caractériser les constituants individuels est élevé. Il est donc essentiel de travailler sur des échantillons qui présentent une taille de plusieurs ordres de grandeur supérieure à la taille des constituants élémentaires, et de considérer que les hypothèses d'homogénéité et d'isotropie au niveau de l'échantillon sont respectées. Ceci permet de garantir la reproductibilité des mesures au niveau de l'échantillon. Les structures anciennes étant très souvent fortement hétérogènes, une représentativité acceptable des matériaux en place nécessitera un échantillonnage adapté.

La manipulation de petits échantillons peut aussi s'avérer délicate dans certains cas, et la fixation souvent problématique sur certains bancs d'essais. Une technique d'enrobage est généralement nécessaire sur les échantillons les plus petits (fig. 3).

2. Projet Comarest - Etude de la compatibilité des matériaux de restauration. Juin 2010 - Mars 2011- Mise en application des résultats du projet Geomat pour éclairer les acteurs de la restauration du Patrimoine quant au choix des matériaux et des techniques à poser dans leur pratique quotidienne en matière de restauration. 

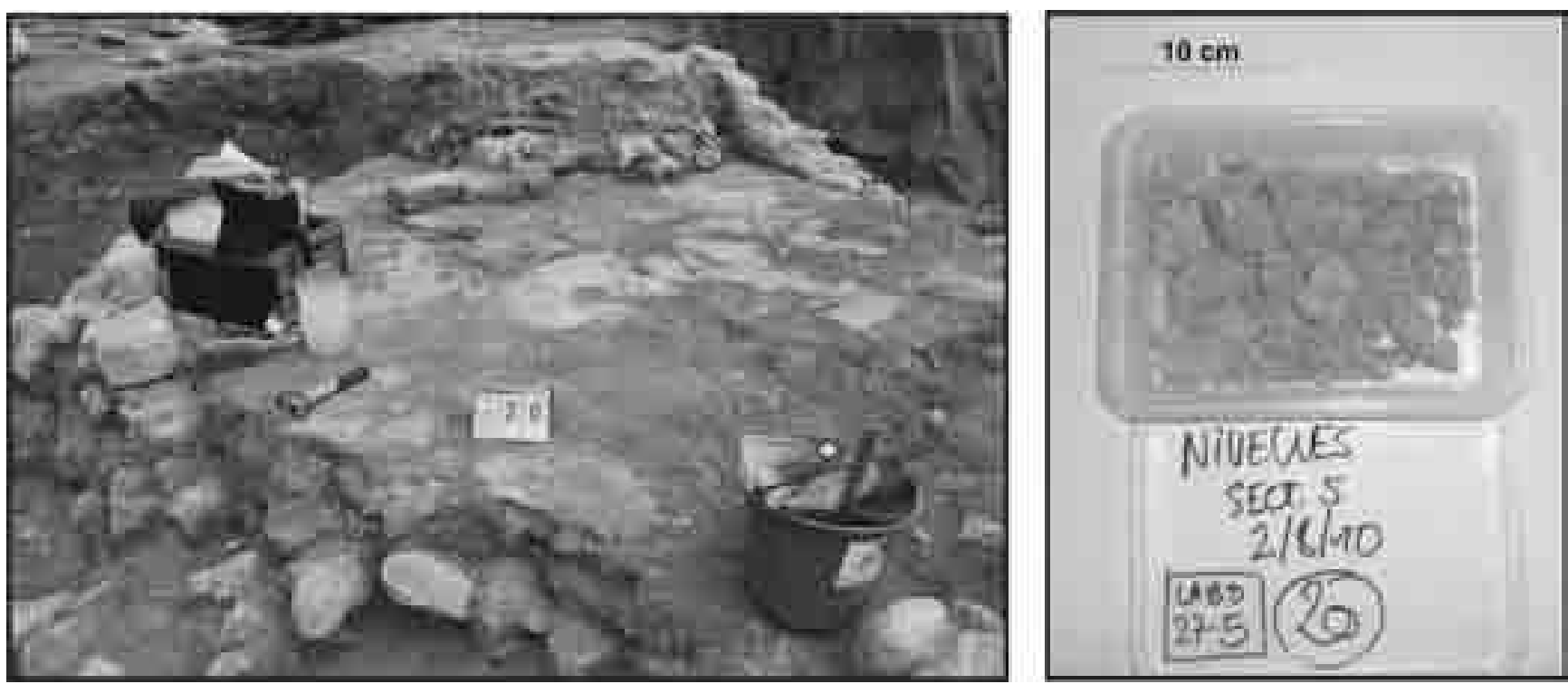

Figure 1 : (Voir planche couleur) Exemple de prélèvement manuel dans les fondations de la Collégiale Sainte-Gertrude à Nivelles (Belgique) à gauche, et le type d'échantillons pouvant faire l'objet de tests de caractérisation à droite.

Figure 1: (See colour plate) Example of manual sampling in the foundations of Collegial Holy-Gertrude in Nivelles (Belgium) on the left, and the type of samples on which characterization tests may be performed on the right.
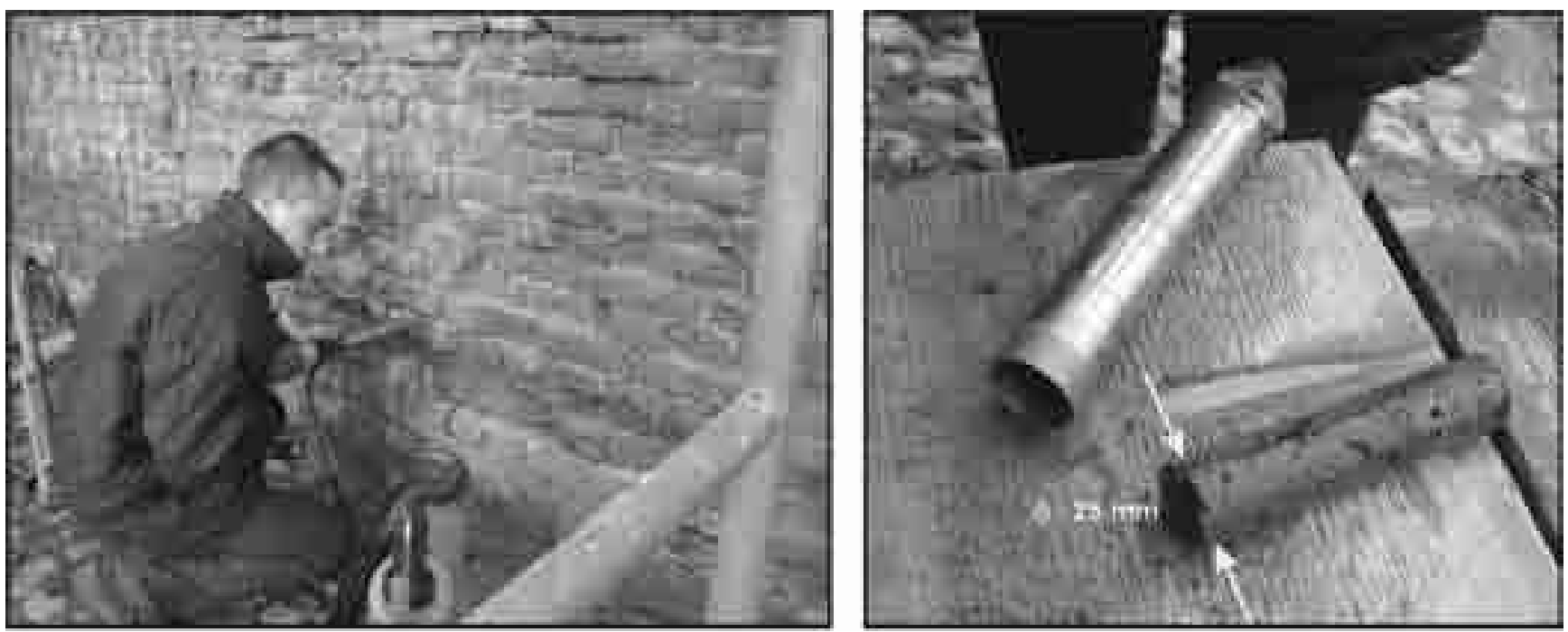

Figure 2 : (Voir planche couleur) Échantillonnage de briques par carottage en petit diamètre avec une machine portative dans les murs de la Ferme de l'Abbaye de Neufvilles (Belgique) à gauche, et exemple d'un prélèvement à droite.

Figure 2: (See colour plate) Brick sampling by small diameter coring with a portable machine in the walls of the Farm of the Abbey of Neufvilles (Belgium) on the left, and example of a sample on the right.

\section{Les procédures de tests}

Les procédures de tests sont reprises succinctement ciaprès dans l'ordre chronologique où ceux-ci sont réalisés. Le lecteur intéressé par plus de détails sur chacune des procédures de tests est invité à se référer à Dagrain et al. (2010a).

\section{Caractérisation de la vitesse sonique}

La vitesse sonique est déterminée à l'aide d'un appareillage classique de type Portable Ultrasonic Non-Destructive Integrity Testing device (PUNDIT, Farnell - fig. 4). L'équipement se compose d'un générateur de train d'ondes 


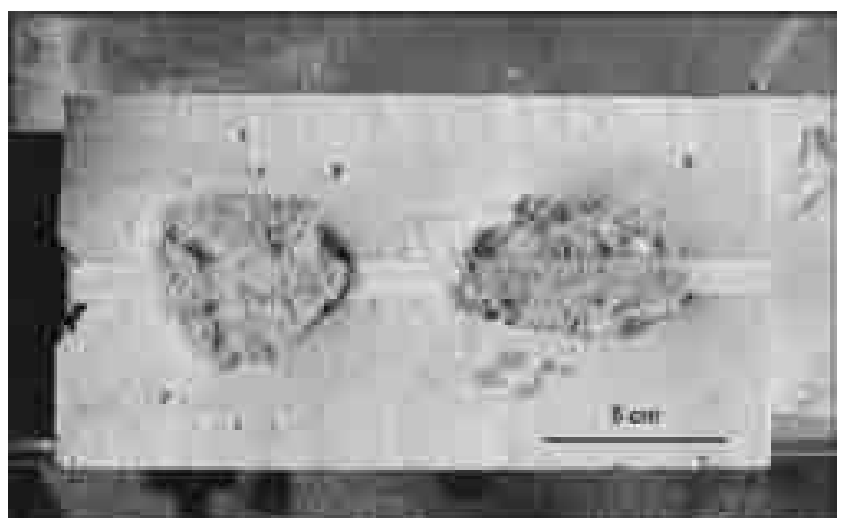

Figure 3 : (Voir planche couleur) Exemple d'enrobage de mortiers historique dans une matrice de plâtre pour permettre la réalisation d'un test de grattage.

Figure 3: (See colour plate) Example of a plaster coating of an historical mortars to allow the realization of a scratching test.

primaires en compression à une fréquence d'émission de $54 \mathrm{kHz}$ et d'un récepteur. L'appareillage détermine le temps de transit mis par le train d'ondes pour se propager de l'émetteur au récepteur (fig. 4).

La technique classique (Rummel et van Heerden, 1978) a été adaptée pour permettre de travailler sur des échantillons de taille réduite. L'utilisation de transducteurs de forme exponentielle à contact ponctuel permet de travailler sur des échantillons de plus petite taille qu'avec les transducteurs traditionnels de $4 \mathrm{~cm}$ de diamètre. De plus, ces transducteurs permettent de travailler sur tous types de surfaces rugueuses, non planes et non parallèles et sans utilisation de fluide de couplage. L'inconvénient majeur de l'utilisation de ces transducteurs sur de petits échantillons est lié au temps de transit, $t_{0}$, que met l'onde pour traverser les deux interfaces transducteurs/échantillons (voir fig. 4) et qui peut être du même ordre de grandeur que le temps réel de transit sur de faibles distances de parcours. Une mesure sur une seule distance de transit peut induire une erreur considérable sur la mesure de la vitesse. La répétition de la mesure du temps de transit sur différentes longueurs permet de s'affranchir de ce problème et de définir précisément la vitesse de propagation des ondes dans les matériaux étudiés.

Les essais se font sur des échantillons bruts récoltés sur le terrain sans préparation préalable, peu importe leur taille et géométrie. Il est cependant important de disposer d'échantillons présentant plusieurs longueurs caractéristiques, de préférence comprises entre 1 et $10 \mathrm{~cm}$. Les essais peuvent être réalisés en mode direct ou indirect selon la géométrie des échantillons. Le test est non destructif, et l'échantillon peut être utilisé par la suite pour d'autres essais.

La connaissance de la valeur de vitesse sonique permet également d'estimer le module de Young dynamique des matériaux, propriété reflétant la rigidité des matériaux, moyennant la connaissance du coefficient de Poisson. Ce coefficient n'est actuellement pas caractérisé par la méthodologie présentée. L'estimation du module de Young dynamique peut donc se faire en utilisant des valeurs du coefficient de Poisson déterminées par essais classiques de compression simple par exemple, ou sont reprises dans la littérature s'il n'est pas possible de déterminer une valeur précise par essais en laboratoire.
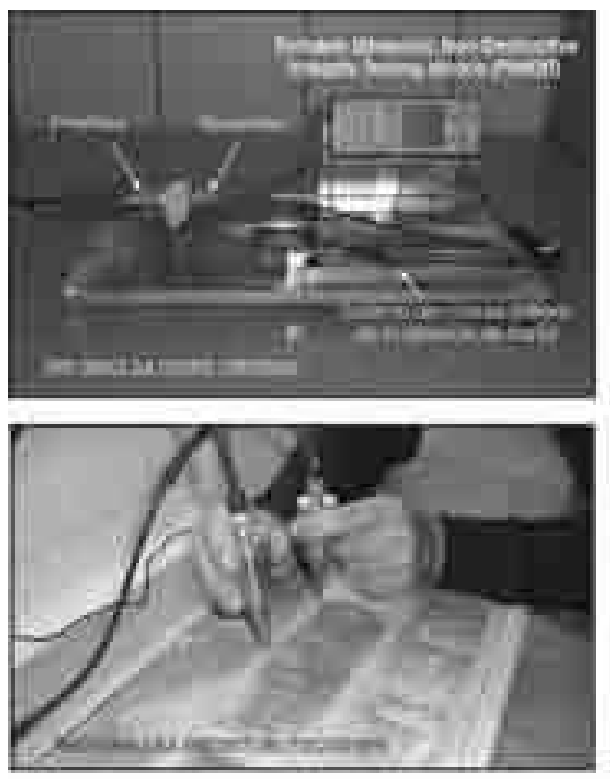

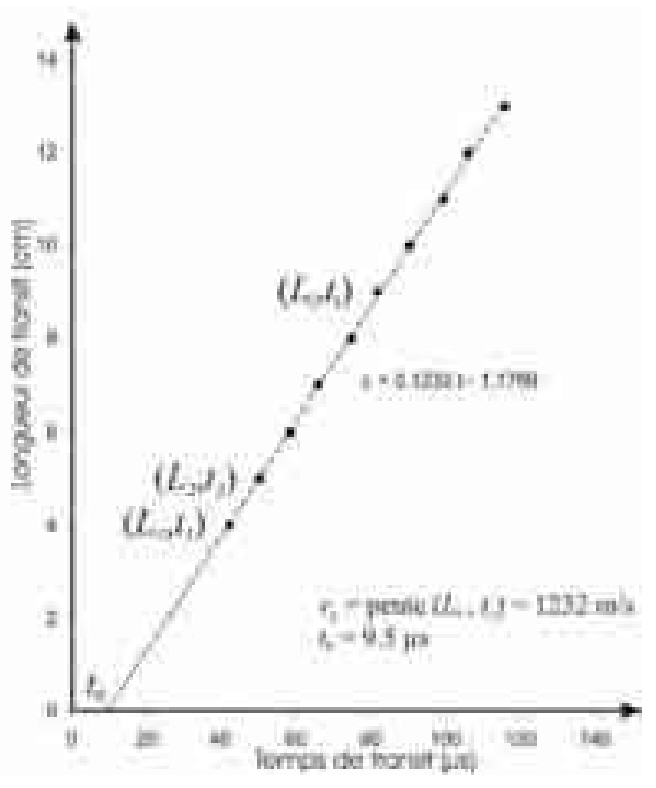

Figure 4 : (Voir planche couleur) Illustration de l'utilisation du PUNDIT en laboratoire sur un banc d'essais adapté pour la réalisation de tests directs (en haut à gauche) ou de tests indirects sur éléments de maçonnerie (en bas à gauche). L'analyse des données se fait en portant en graphique les distances de transit en fonction du temps de parcourt (à droite). Figure 4: (See colour plate) Illustration of the use of the PUNDIT in laboratory on a testing bench adapted for the realization of direct tests (in top on the left) or indirect test on elements of masonry (in bottom on the left). The data analysis is done by plotting the transit distances versus the transit time (on the right). 


\section{Caractérisation de la densité et de la porosité ouverte}

La densité apparente et la porosité d'un échantillon sont des propriétés qui nécessitent une mesure précise du volume total, aussi appelé volume enveloppe, et du volume des pores. La mesure du volume enveloppe se fait via l'utilisation d'un volumètre à flux de particules solides (Geopyc 1360, Micromeritics - fig. 5) (Webb, 2001). Le volume des pores quant à lui ne se mesure pas directement, mais est déterminé par la différence entre le volume enveloppe et le volume structurel d'un même échantillon. Le volume structurel est mesuré précisément $\left(10^{-3} \mathrm{~cm}^{3}\right)$ à l'aide d'un pycnomètre à gaz (Accupyc 1340, Micromeritics - fig. 5). L'intérêt de la méthode comparativement aux méthodes classiques est de pouvoir travailler sur des échantillons de taille très réduite (de l'ordre du $\mathrm{cm}^{3}$ et de maximum $25 \mathrm{~cm}^{3}$ ), de forme pouvant être quelconque, préalablement séchés, et sans devoir réaliser de phase de saturation à l'eau qui peut être critique dans le cas de matériaux très sensibles à l'eau. La méthode est excessivement rapide comparativement aux méthodes classiques (20 minutes). La détermination de la densité nécessite en plus l'utilisation d'une balance de précision (ALJ 160, Kern, à $10^{-4} \mathrm{~g}-$ fig. 5). Les tests sont non-destructifs.

\section{Caractérisation du coefficient de capillarité}

Le coefficient de capillarité, aussi appelé coefficient de remontée capillaire [Beck et al. 2003, Carré et al.2005], est déterminé à l'aide d'une balance de précision (ALJ 160, Kern, à $10^{-4} \mathrm{~g}$ ) mesurant le gain en masse d'un échantillon suspendu à un crochet sous balance, et plongé partiellement sous une hauteur d'eau de deux millimètres (fig. 6).
La balance est raccordée à un ordinateur d'acquisition des données qui permet d'enregistrer la courbe de remontée capillaire de manière continue sans manipulation de l'échantillon comme dans les méthodes classiques. La partie linéaire de la courbe du gain massique surfacique en fonction de la racine carrée du temps permet de déterminer le coefficient de capillarité $\left[\mathrm{gr} / \mathrm{m}^{2} / \mathrm{s}^{1 / 2}\right]$. Le test se fait avec de l'eau distillée, ou tout autre type de fluide selon les applications, dont notamment dans le cas de traitement de matériaux par fluides imperméabilisant ou durcisseurs. La mesure de la capillarité étant en fait une mesure de débit de fluide à travers la section de l'échantillon au contact de l'eau, l'aire de celle-ci doit être mesurable et donc présenter une forme simple et plane. Le test se fait de préférence sur un échantillon de forme cylindrique de 18 ou $25 \mathrm{~mm}$ de diamètre. Le test est non destructif, mais nécessite la préparation d'un cylindre qui est généralement utilisé pour les mesures de densité et porosité.

\section{Caractérisation de la dureté Leeb}

Le test de dureté Leeb consiste à effectuer une mesure de dureté à l'aide d'un scléromètre de type Shore utilisant un corps impactant muni d'une bille en carbure de tungstène de $3 \mathrm{~mm}$ de diamètre et de dureté $1500 \mathrm{HV}$ (appareil Equotip Piccolo de chez Proceq - fig. 7). La mesure réalisée lors du test permet de chiffrer la perte d'énergie cinétique du corps impactant lors de son rebond après avoir percuté la surface du matériau à caractériser. Cette perte d'énergie cinétique est déterminée par la mesure des vitesses du corps impactant avant et après impact. La mesure de dureté Leeb n'est rien
Figure 5: Illustration des équipements utilisés pour la détermination de la densité apparente et de la porosité ouverte.

Figure 5: Illustration of the equipment used for the determination of the apparent density and open porosity.
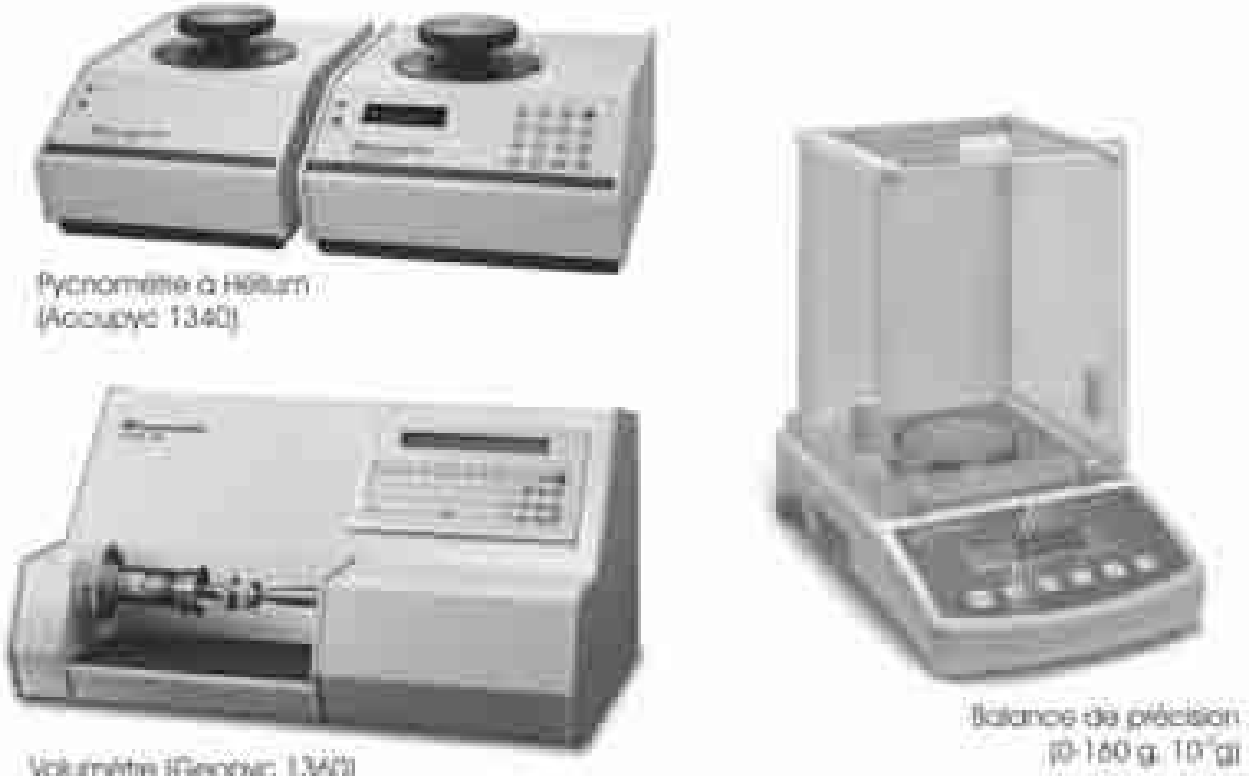


\section{Essai de capillarite dans une plerre calcaire poreuse (41\%)}

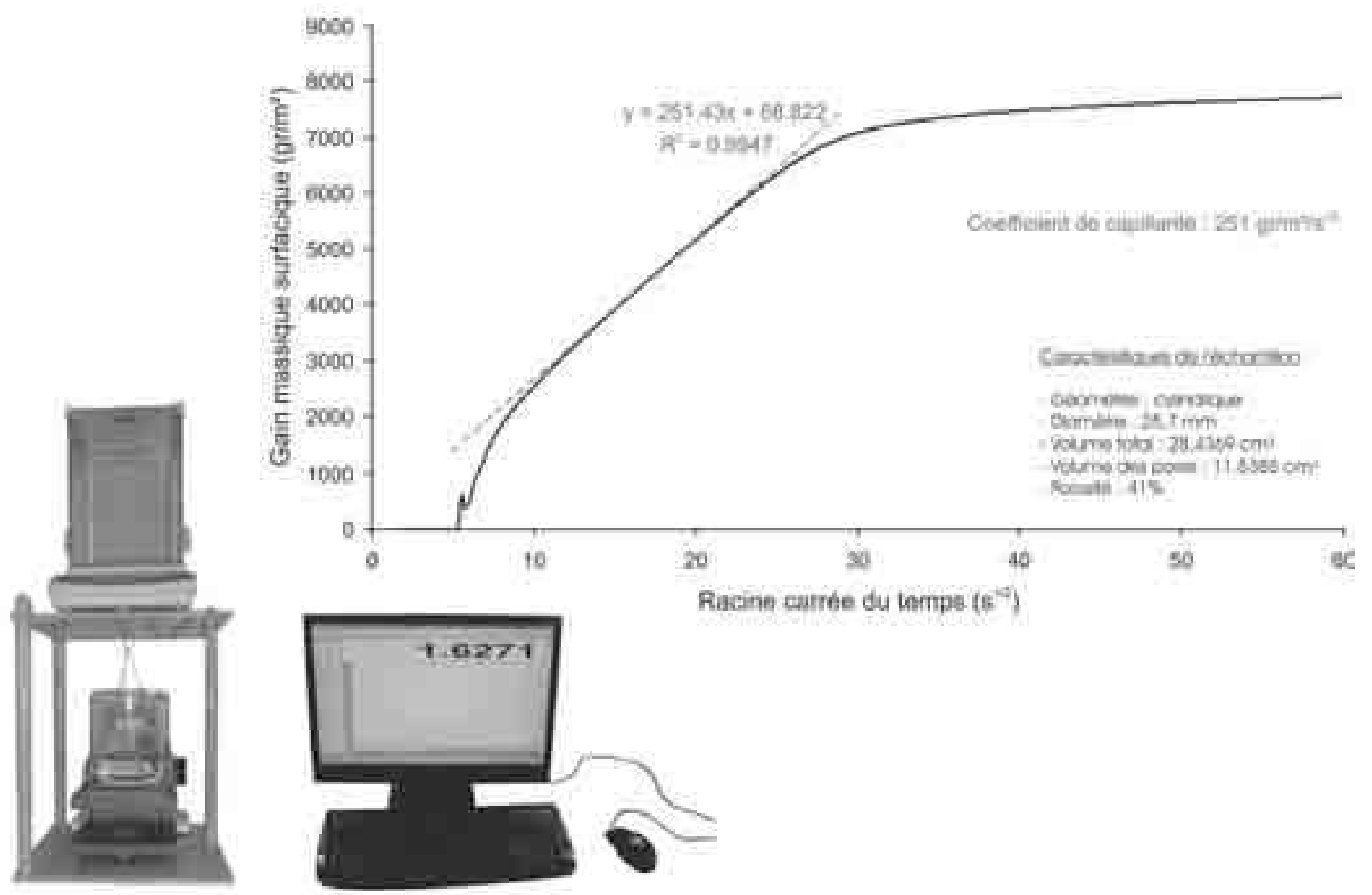

Figure 6 : Illustration de l'utilisation de la balance de précision pour déterminer le coefficient de capillarité. L'analyse des données se fait en portant en graphique le gain massique surfacique en fonction de la racine carrée du temps. Le coefficient de capillarité est la pente de la partie linéaire de la courbe.

Figure 6: Illustration of the use of the accurate balance to determine the capillarity coefficient. The data analysis is done by plotting the increase of mass versus the square root of time. The coefficient of capillarity is the slope of the linear part of the curve.

d'autre que le rapport des deux vitesses multiplié par 1000 (Leeb, 1986).

Les principales limitations de ce type de test sur de petits échantillons résident dans les problèmes de fixation des échantillons sur un support rigide et dans la fragilité de l'échantillon à tester. Ces limitations ont été résolues par la mise au point d'une technique d'enrobage non invasif permettant de maintenir les échantillons de petite taille sur un support massif ou de les fixer dans un étau rigide (fig. 7).

La procédure de test consiste à effectuer 10 rebonds au même endroit, et de répéter la mesure à différents emplacements sur l'échantillon. Il est important de signaler que la succession des rebonds au même endroit provoque une compaction de la matière telle que la mesure de dureté en est influencée. Il y a donc lieu de faire la distinction entre la dureté de premier rebond représentative du matériau initial et la dureté asymptotique caractéristique du matériau compacté par le corps impactant. Si la dureté de premier rebond est directement liée aux propriétés élastiques de la matière, la différence entre les duretés asymptotique et de premier rebond est quant à elle liée à la porosité du matériau. La mesure de dureté étant une valeur ponctuelle, il est recommandé de réaliser un maximum de mesures pour avoir une bonne représentativité de la mesure à l'échelle de l'échantillon.

La caractérisation se fait sur tout type d'échantillons présentant un volume supérieur au $\mathrm{cm}^{3}$, et de préférence sur une surface plane dans la mesure du possible. L'intérêt de la méthode est d'accéder facilement à la mesure de la résistance des matériaux à l'aide d'un test simple, très rapide, et non 
Figure 7 : Illustration du montage expérimental et méthode d'analyse des résultats d'essais. Les échantillons peuvent être maintenus dans un petit étau ou enrobé dans une matrice dont le liant est adapté au type de matériaux à étudier. Figure 7: Illustration of the testing bench and the data analysis method. The samples can be maintained in a small vice or coated in a matrix whose binder is adapted to the type of materials to study.
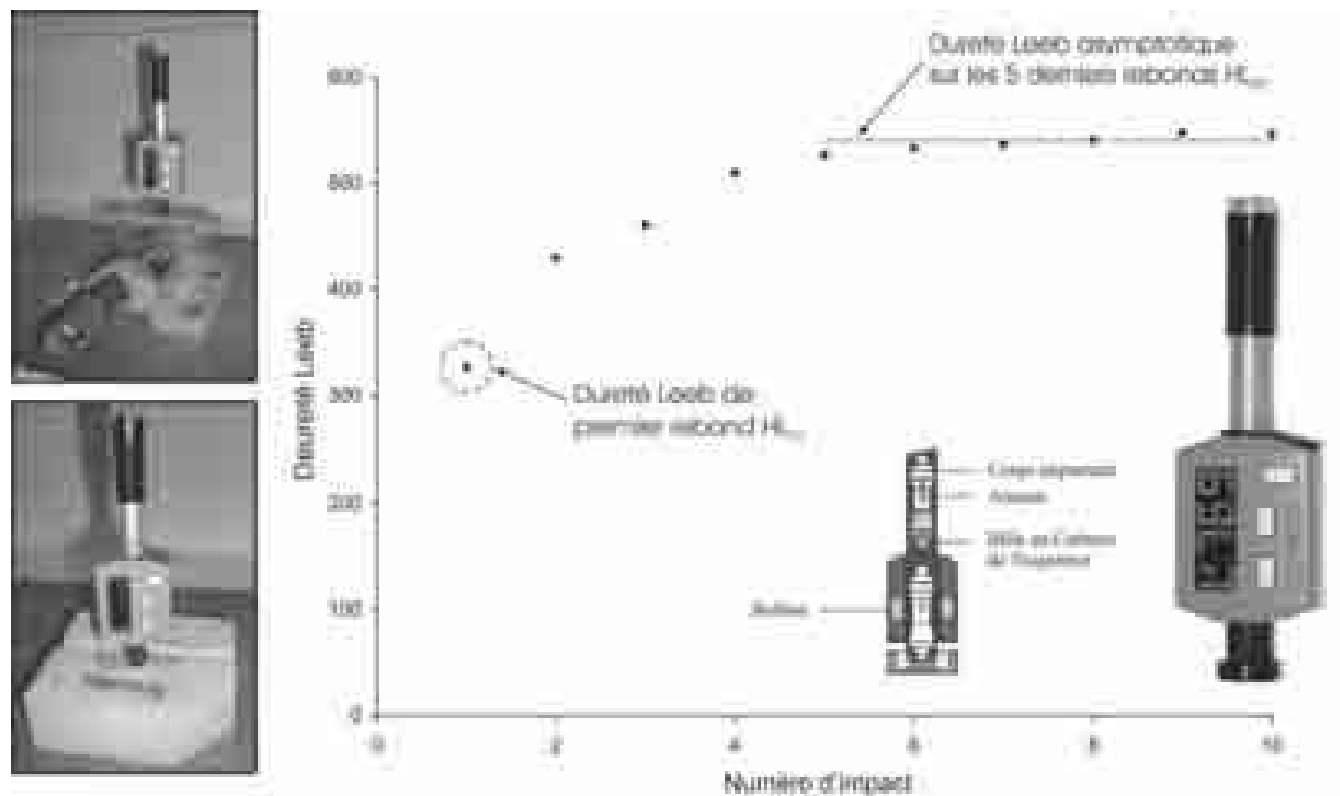

destructif. Verwaal et Mulder (1993) ont ainsi établi une corrélation entre résistance en compression et dureté Leeb de premier rebond pour les pierres naturelles présentant des résistances variant de 5 à $200 \mathrm{MPa}$.

\section{Caractérisation de l'énergie spécifique intrinsèque et de l'angle de frottement}

L'énergie spécifique intrinsèque et l'angle de frottement d'un matériau peuvent être déterminés à l'aide d'un test de grattage (Dagrain, 2007). Ce test consiste à éliminer une couche superficielle du matériau à caractériser à l'aide d'un outil de coupe diamanté (fig. 8). Le test est répété pour différentes profondeurs de passe variant de $50 \mu \mathrm{m}$ à $1 \mathrm{~mm}$. Les propriétés mécaniques des matériaux influencent directement les mécanismes de destruction et l'amplitude des efforts générés au niveau de l'outil lors du test de grattage. La mesure des efforts sur l'outil diamanté se fait de manière continue durant le test, et l'analyse des efforts de coupe $a$ posteriori permet d'en caractériser (i) l'énergie spécifique intrinsèque qui est l'énergie volumique de destruction du matériau $\left[\mathrm{mJ} / \mathrm{mm}^{3}\right.$ ou $\mathrm{MPa}$ ] et (ii) l'angle de frottement entre l'outil et le matériau analysé. Il a été mis en évidence dans plusieurs publications scientifiques que la mesure de l'énergie spécifique intrinsèque est parfaitement corrélée à la mesure de la résistance en compression simple des matériaux (Dagrain et al., 2006), et l'angle de frottement en coupe est égal à l'angle de frottement interne des matériaux (Dagrain et Richard, 2006).

\section{Caractérisation de la résistance par micro-forage}

Le test de micro-forage est assez similaire au test de grattage [Dagrain et Descamps, 2010] et les concepts théoriques de base sont strictement identiques. Le test de micro-forage consiste en la destruction par forage de matériaux à l'aide d'un outil diamanté de $5 \mathrm{~mm}$ de diamètre sur des profondeurs pouvant aller jusque $8 \mathrm{~cm}$. Un appareillage commercial, le Drilling Resistance Measuring System (DRMS), a été spécialement développé pour mesurer in situ la résistance au forage de matériaux pierreux [Fratini et al., 2006] et de mortiers [Delmonte et Vignoli, 2008]. Le DRMS présentant un certain nombre de limitations techniques, le choix s'est porté pour le développement d'une perceuse automatisée et instrumentée en laboratoire (fig. 9). Le test est répété pour différentes vitesses d'avance variant de 50 à 300 micromètres par tour. Les vitesses de rotation $(250 \mathrm{tr} / \mathrm{min})$ et d'avance sont maintenues constantes durant le test. La force de poussée et le couple au niveau de la mèche, sont enregistrés en continu durant le forage. Si l'analyse des résultats d'essais obtenus avec le DRMS se fait en termes de résistance au forage (poussée moyenne rapportée à la section du trou foré [MPa]), l'analyse des données d'essais sur la perceuse instrumentée se fait, de manière similaire au test de grattage, en portant en graphique le couple en fonction de la vitesse d'avance de la mèche. Pour des essais en laboratoire, un échantillon de quelques $\mathrm{cm}^{3}$ est déjà amplement suffisant. Plusieurs publications scientifiques ont été rédigées pour illustrer la forte corrélation existant entre la résistance au forage et les propriétés mécaniques des matériaux et notamment la résistance en compression simple [Fernandes 

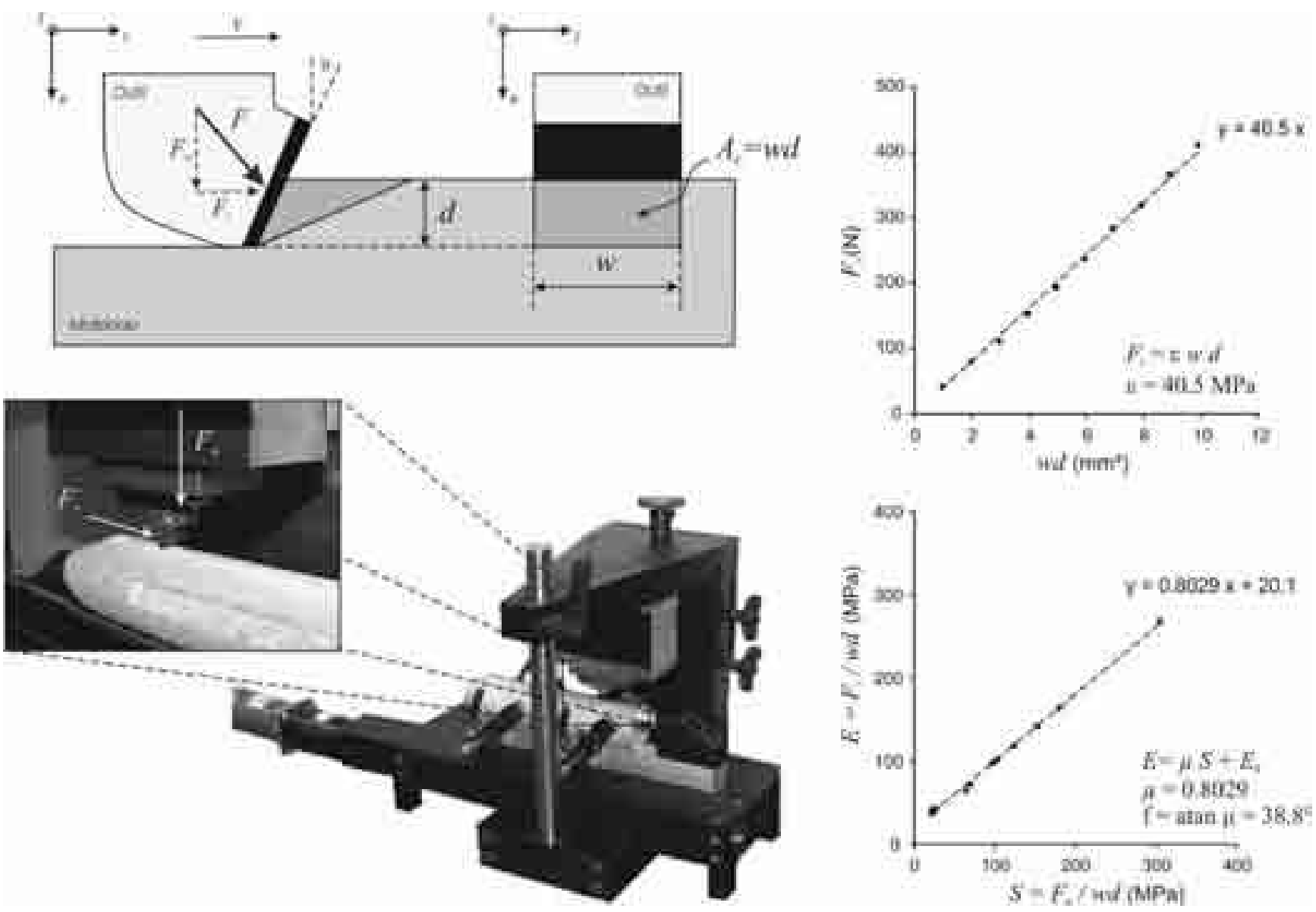

Figure 8 : (Voir planche couleur) Illustration d'un banc d'essais de grattage. L'analyse des données se fait tout d'abord en portant en graphique la force tangentielle en fonction de la section active de l'outil $(w d)$. La pente de la droite de régression est définie comme étant l'énergie spécifique intrinsèque $(\varepsilon)$. Ensuite, les rapports $F \mathrm{t} / w d$ et $F \mathrm{n} / w d$ peuvent aussi être portés en graphique. La pente de la droite de régression permet d'accéder au coefficient de frottement interne du matériau $(\mu)$.

Figure 8: (See colour plate) Illustration of the scratching device. The data analysis is done first of all by plotting the tangential force versus the cross sectional area of the cut $(w d)$. The slope of the straight regression line is defined as being the intrinsic specific energy $(\varepsilon)$. Then, the Ft/wd and Fn/wd ratios can also be plotted. The slope of the straight regression line gives access to the internal coefficient of friction of the material $(\mu)$.

et Lourenço, 2007; Pamplona et al., 2007] mais dans des conditions de fonctionnement déterminées (RPM $600 \mathrm{tr} /$ min et ROP $10 \mathrm{~mm} / \mathrm{min}$ ). Les travaux de recherche menés actuellement montrent que l'utilisation de la méthode de test utilisée sur la perceuse de laboratoire confirme cette tendance mais indépendamment des conditions de fonctionnement imposées.

\section{Validation par recoupement}

Les diverses propriétés déterminées avec la méthodologie mise au point peuvent être croisées entre-elles pour vérifier leur validité. Une base de données recueillant bon nombre de ces propriétés sur une multitude de matériaux différents a permis de mettre en évidence des tendances entre les diverses propriétés. L'utilisation de ces tendances permet ainsi, par exemple, de détecter une propriété présentant une valeur " anormale ", et de recommander la réalisation d'un test de vérification. Dans certains cas, si un ou plusieurs tests de la méthodologie ne peuvent être réalisés sur les échantillons de plus petites tailles, la base de données peut être utilisée, mais avec précaution, pour obtenir une estimation des propriétés manquantes moyennant l'usage des corrélations.

\section{Types de matériaux}

Tous types de matériaux de construction peuvent être analysés avec la méthodologie mise au point : pierres naturelles, 

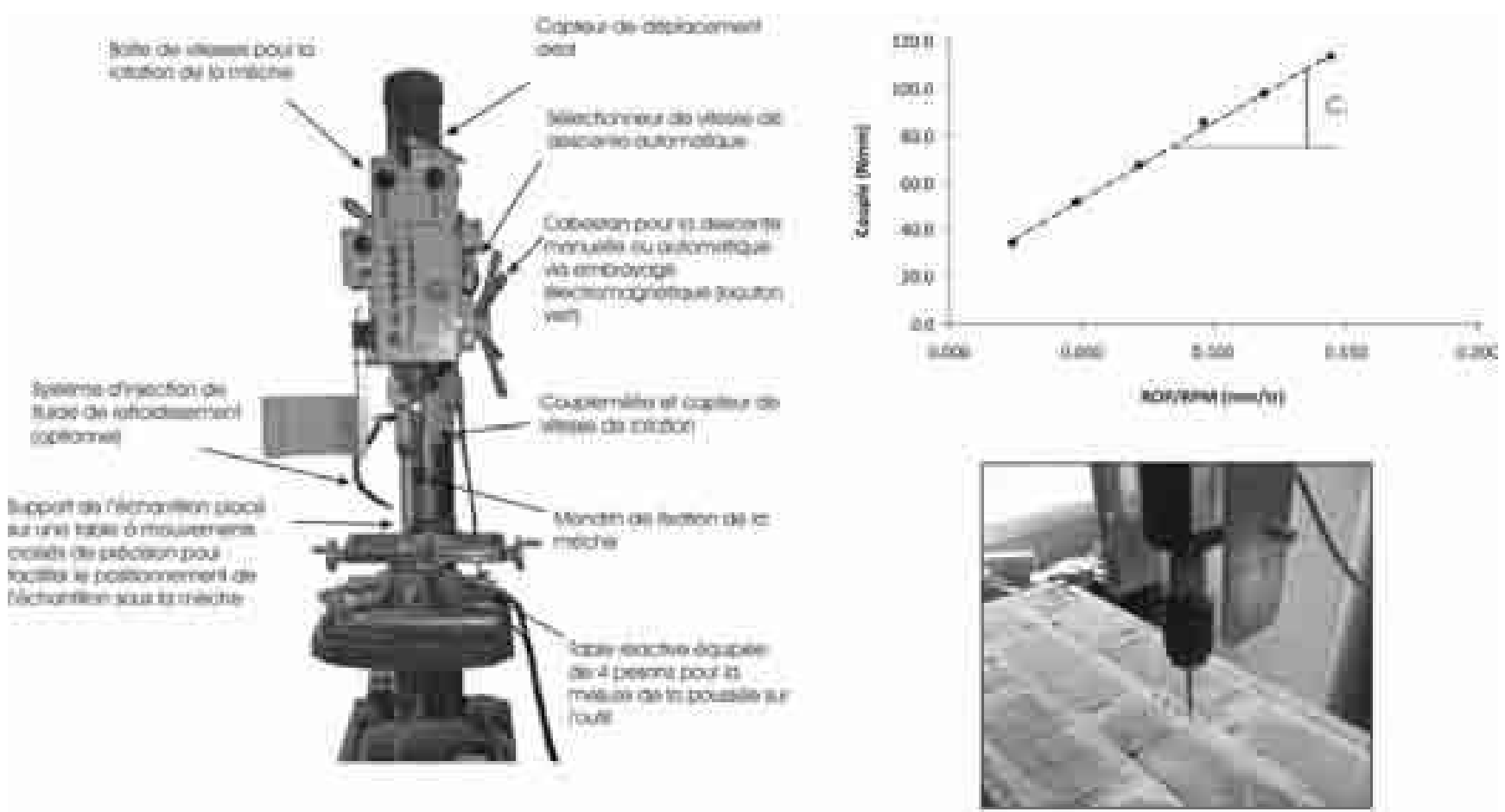

Figure 9: (Voir planche couleur) Illustration du banc d'essais de micro-forage et exemple d'application dans un joint de mortier d'une maçonnerie. L'analyse des données se fait en portant en graphique le couple en fonction de la vitesse d'avance de la mèche (ROP/RPM en $\mathrm{mm} / \mathrm{tr}$ ). Comme pour le test de grattage, la pente de cette courbe est directement proportionnelle à la résistance du matériau foré.

Figure 9: (See colour plate) Illustration of the micro-drilling device and example of application in a joint of mortar of a masonry. The data analysis is done by plotting the torque on bit versus the rate of penetration of the bit (ROP/RPM in mm/tr). As for the test of scratching test, the slope of this curve is directly proportional to the strength of drilled material.

mortiers, briques et tuiles en terre cuite, enduits... Les matériaux sont tous caractérisés en suivant les mêmes procédures expérimentales. Les propriétés physico-mécaniques de divers types de matériaux peuvent ainsi être comparées directement sans utilisation de règles particulières de conversion tenant compte des normes d'essais suivies, des tailles et formes des échantillons.

\section{EXEMPLES D'APPLICATION DE LA MÉTHODOLOGIE}

La méthodologie a été développée initialement pour la caractérisation de matériaux rocheux rencontrés dans des travaux de génie civil, de forage pétrolier à très grandes profondeurs, ou de travaux de dragage maritime et d'enrochement de digues. Cette méthodologie a fait ses preuves et est régulièrement mise en application. Un transfert de technologie a été effectué vers le domaine de la construction afin d'utiliser cette méthodologie pour la caractérisation des propriétés mécaniques sur divers types de matériaux de construction du patrimoine bâti. Nous reprenons ci-dessous une synthèse des exemples les plus parlants.

\section{Étude des mortiers historiques de la Tour Henry VIII à Tournai}

La tour de Henry VIII (fig. 10) a été construite au $\mathrm{XVI}^{\mathrm{e}}$ siècle, sous le Roi Henry VIII Tudor. Cette tour est le seul vestige qui subsiste de la forteresse anglaise située à Tournai, Belgique [Dosogne, 2009; Deramaix et Dosogne, 2009]. Cette tour était la première structure militaire réalisée après la première apparition de l'artillerie à poudre. Son diamètre est de 27,5 mètres, et sa hauteur est de 20 mètres. En 1562, la structure a été employée comme prison et est devenue un musée militaire au XIX ${ }^{\mathrm{e}}$ siècle. La tour a été restaurée en 1852. En 1963, la tour était répertoriée comme monument par les autorités. En 2007 et 2008, des enquêtes préliminaires ont été réalisées préalablement à de nouveaux travaux de restauration. Dans ce contexte et en collaboration avec les archéologues responsables du chantier, des échantillons de mortiers historiques ont été prélevés dans 


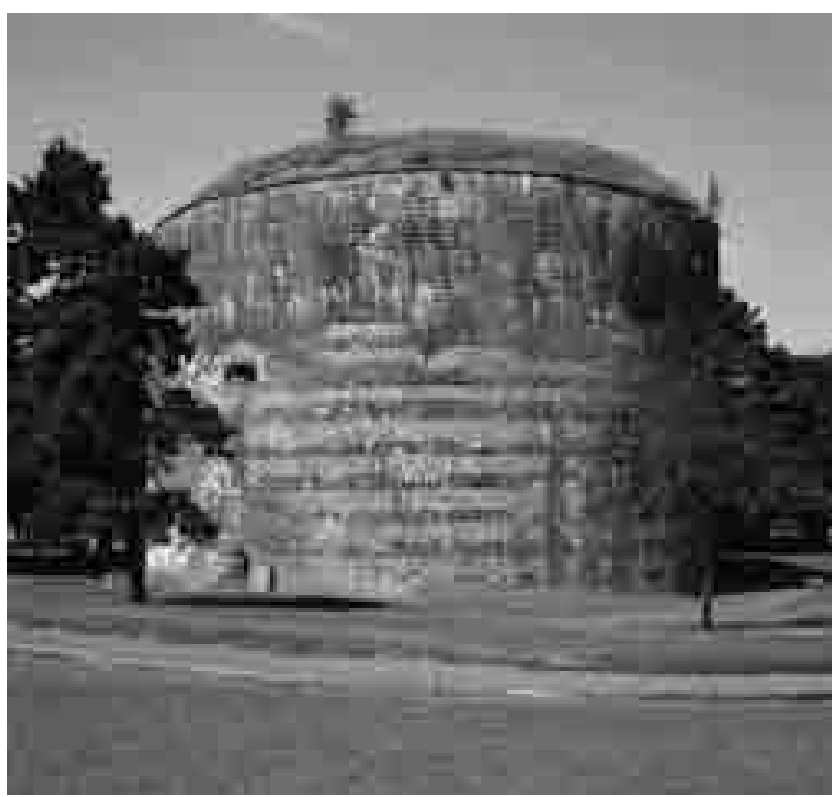

Figure 10 : (Voir planche couleur) Tour Henry VIII à Tournai, Belgique (Guy Focant (C) SPW).

Figure 10: (See colour plate) Henry VIII Tower in Tournai, Belgium (Guy Focant (C) SPW). la structure et analysés sur base de la méthodologie proposée. L'objectif de ce programme de recherche était essentiellement de vérifier la faisabilité de la méthodologie pour déterminer les propriétés mécaniques des mortiers historiques à partir d'échantillons pouvant être de taille très réduite, de très faible résistance ou peu consolidés. Quelques exemples d'échantillons prélevés sur le site de la Tour Henry VIII sont illustrés en figure 11 après essais. La trace de la rainure de l'essai de grattage et les trous des essais de micro-forage sont ainsi visibles sur certains échantillons. Les diverses propriétés physico-mécaniques déterminées sont reprises dans le tableau 1. Les résultats de cette étude ont fait l'objet d'un rapport détaillé non publié [Demellenne, 2010].

Les valeurs d'énergie spécifique intrinsèque observées semblent relativement élevées pour des mortiers historiques. En effet, d'une manière générale, les projets Geomat et Comarest avaient mis en évidence que les mortiers de chaux utilisés actuellement en restauration du patrimoine étaient caractérisés par des énergies spécifiques intrinsèques bien en dessous de $10 \mathrm{MPa}$, tandis que les pierres naturelles présentes dans les maçonneries anciennes pouvaient avoir des énergies spécifiques variant de 10 à 100 voire $150 \mathrm{MPa}$. Les

\section{Echantillon N 39}

Echantilion N337

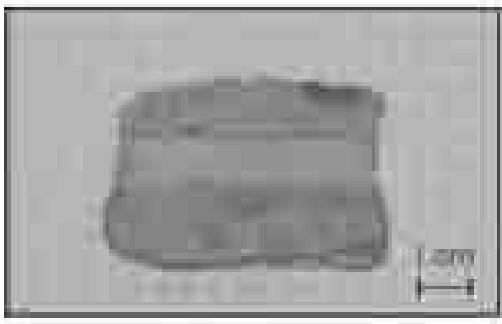

Echantilion $\mathrm{N}^{3} 38$

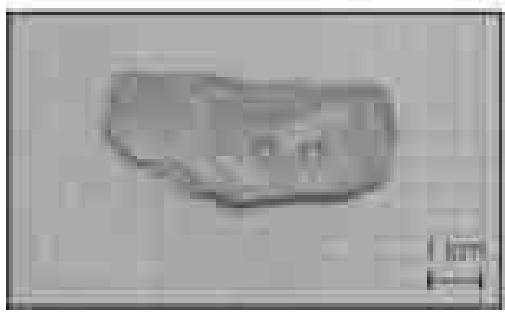

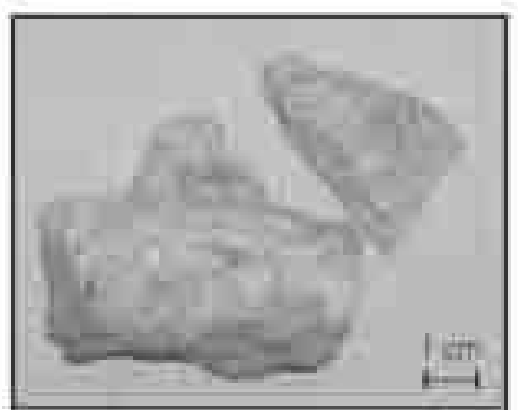

Echantillon $\mathrm{N}^{2} 40$

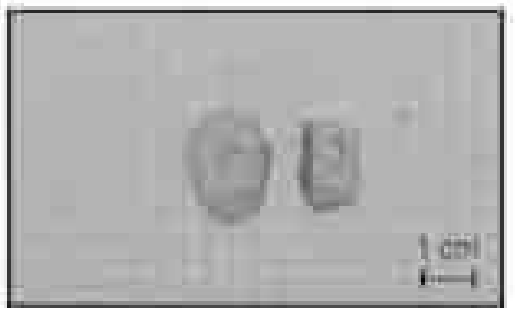

Echantillon N 42

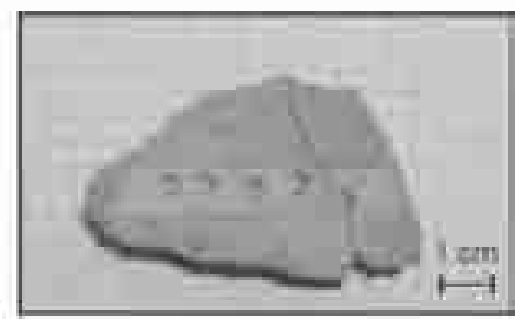

Echantillon N/44

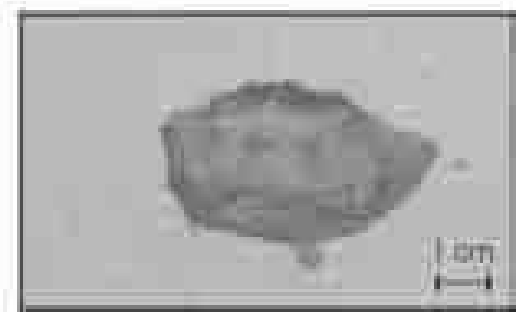

Figure 11 : (Voir planche couleur) Quelques exemples d'échantillons prélevés sur la Tour Henry VIII et testés avec la méthodologie présentée. La rainure laissée par le test de grattage et les trous des essais de micro-forage sont visibles sur plusieurs des échantillons. À noter la taille assez réduite des deux petits morceaux testés pour l'échantillon numéro 40, non testable par le test de grattage.

Figure 11: (See colour plate) Some examples of samples taken on the Henry VIII Tower and tested with the methodology presented. The groove left by the scratching test and the holes of the micro-drilling tests are visible on several of the samples. To note the size rather reduced of the two small pieces tested for sample number 40 , not testable with the scratching test. 


\begin{tabular}{|c|c|c|c|c|c|c|c|c|c|}
\hline \multirow{2}{*}{ Réf. } & \multirow{2}{*}{ Age } & \multirow{2}{*}{ US } & \multirow{2}{*}{ Description } & \multirow{2}{*}{ Couleur } & \multirow{2}{*}{$\mathrm{d}$} & $\phi$ & $\mathrm{Vp}$ & $\varepsilon$ & $\mathrm{Dr}$ \\
\hline & & & & & & $\%$ & $\mathrm{~m} / \mathrm{s}$ & $\mathrm{MPa}$ & $\mathrm{MPa}$ \\
\hline 35 & $\mathrm{XIX}^{\mathrm{e}}$ & US 029 & $\begin{array}{l}\text { réaménagement maçonnerie en esca- } \\
\text { lier au sommet du monument }\end{array}$ & gris & 1.98 & 27.2 & 1977 & 25.84 & 0.868 \\
\hline 36 & $\mathrm{XIX}^{\mathrm{e}}$ & US 029 & $\begin{array}{l}\text { mortier sous le larmier } \mathrm{XIX}^{\mathrm{e}} \text { au som- } \\
\text { met du monument }\end{array}$ & $\begin{array}{l}\text { gris avec des } \\
\text { plages rouille }\end{array}$ & 1.40 & 41.5 & 917 & 20.48 & 0.346 \\
\hline 37 & & US080 & murenbr iqueca ged 'escalier & $\begin{array}{l}\text { grisa vec } \\
\text { fragments } \\
\text { blanchâtres }\end{array}$ & 1.72 & 17.4 & 1996 & 43.74 & 0.654 \\
\hline 38 & s.d. & US026 & $\begin{array}{l}\text { parement extérieur RDC gros blocs } \\
\text { assisés -liaison des blocs du parement }\end{array}$ & $\begin{array}{l}\text { gris- blanc avec } \\
\text { petits cailloux } \\
\text { blancs? }\end{array}$ & 1.61 & 23.4 & 1704 & 17.06 & 0.575 \\
\hline 39 & $\mathrm{XVI}^{\mathrm{e}}$ & US 004 & $\begin{array}{l}\text { milieu de carottage du mur de } 7 \mathrm{~m} \\
\text { d'épaisseur au } 1^{\text {er étage }}\end{array}$ & $\begin{array}{l}\text { blanc avec agré- } \\
\text { gats lithiques }\end{array}$ & 1.45 & 43.3 & 1735 & 10.72 & 0.214 \\
\hline 40 & & US092 & $\begin{array}{l}\text { mur en brique au débouché de } \\
\text { l'escalier }\end{array}$ & $\begin{array}{l}\text { gris avec frag- } \\
\text { ments blancs } \\
\text { (pierres?) }\end{array}$ & 1.57 & 33.7 & 1335 & - & 0.373 \\
\hline 41 & & US004 & $\begin{array}{l}\text { parement extérieur RDC gros blocs } \\
\text { assisés intérieur blocage }\end{array}$ & blancg risâtre & 1.39 & 47.2 & 1175 & 10.10 & 0.066 \\
\hline 42 & $\mathrm{XIX}^{\mathrm{e}}$ & US029 & $\begin{array}{l}\text { réaménagement maçonnerie en esca- } \\
\text { lier au sommet du monument }\end{array}$ & gris & 1.78 & 26.4 & 2239 & 43.59 & 0.555 \\
\hline 43 & $\mathrm{XVI}^{\mathrm{e}}$ & US 097 & $\begin{array}{l}\text { mur en pierre calcaire de Tournai au } \\
\text { débouché de l'escalier }\end{array}$ & $\begin{array}{l}\text { gris avec no- } \\
\text { dules blancs }\end{array}$ & 1.67 & 25.0 & 1936 & 29.80 & 0.734 \\
\hline 44 & $\mathrm{XIX}^{\mathrm{e}}$ & US094 & Blocagesi milaireà l ’USO 29 & grisbr un & 1.99 & 17.3 & 2182 & 29.70 & 0.601 \\
\hline 45 & $\mathrm{XIX}^{\mathrm{e}}$ & US 029 & $\begin{array}{l}\text { Mur au débouché de l'escalier } \\
\text { coffrage }\end{array}$ & $\begin{array}{l}\text { gris brun ou } \\
\text { gris blanc }\end{array}$ & 1.87 & 9.2 & 1354 & 32.58 & 0.819 \\
\hline
\end{tabular}

Tableau 1 : Propriétés déterminées sur des mortiers historiques prélevés dans la Tour Henry VIII. $d:$ densité $-\phi:$ porosité - Vp : vitesse sonique $-\varepsilon$ : énergie spécifique intrinsèque $-D \mathrm{r}$ :r ésistancea uf orage.

Table 1: Properties determined on historical mortars sampled on the Henry VIII Tower.

valeurs les plus basses, aux alentours de $10 \mathrm{MPa}$, concernent probablement les échantillons les plus anciens : le milieu de carottage du mur de 7 mètres d'épaisseur au $1^{\text {er }}$ étage $\left(n^{\circ} 39\right)$ et l'intérieur du blocage de gros blocs assisés du parement extérieur du rez-de-chaussée ( $\left.{ }^{\circ} 41\right)$, qui correspondent tous deux à la construction d'origine, datant du XvI ${ }^{\mathrm{e}}$ siècle. Une exception notoire est celle du ${ }^{\circ} 43$, qui se rapporte à une unité stratigraphique datée par les archéologues du $\mathrm{XVI}^{\mathrm{e}}$ siècle et qui présente une valeur d'énergie spécifique intrinsèque importante, approchant les $30 \mathrm{MPa}$. Les valeurs d'énergie spécifique intrinsèque les plus élevées se rapportent aux unités stratigraphiques datées du XIx ${ }^{e}$ siècle et dont les observations macroscopiques, révélant une couleur grise caractéristique, semblent indiquer, sous réserve d'analyses chimiques, la présence de ciment.

Les échantillons les plus poreux sont ici aussi liés aux unités stratigraphiques probablement les plus anciennes: le milieu de carottage du mur de 7 mètres d'épaisseur au $1^{\text {er }}$ étage ( $\left.n^{\circ} 39\right)$ et l'intérieur du blocage de gros blocs assisés du parement extérieur du rez-de-chaussée ( $\left.n^{\circ} 41\right)$. Les échantillons provenant du coffrage du mur au débouché de l'escalier $\left(\mathrm{n}^{\circ} 45\right)$ et du mur en brique de la cage d'escalier $\left(n^{\circ} 37\right)$, ainsi que le $n^{\circ} 44$ sont quant à eux peu, voire très peu poreux $\left(\mathrm{n}^{\circ} 45\right)$. Ces trois prélèvements sont tous datés du XIx ${ }^{e}$ si ècle.

Une corrélation entre la résistance et la porosité semble ressortir de l'analyse des résultats (voir fig. 12). Cette corrélation semble en plus liée à l'âge des compositions étudiées : la résistance diminue, tandis que la porosité augmente avec l'âge des mortiers. L'hypothèse ainsi formulée, en vertu de laquelle plus la composition est ancienne, plus elle est poreuse et moins elle est résistante devrait dans la mesure du possible guider la sélection des formulations pour la conservation - restauration du monument.

Les résultats de cette étude de faisabilité ont permis d'initier la mise en application de cette méthodologie de caractérisation dans le cadre d'une recherche doctorale ayant pour but l'étude des mortiers historiques en termes de chronologie et d'applications structurales et fonctionnelles [Demelenne, 2011]. À plus long terme, la méthodologie devrait pouvoir être appliquée pour la caractérisation des mortiers historiques dans les bâtiments patrimoniaux afin 


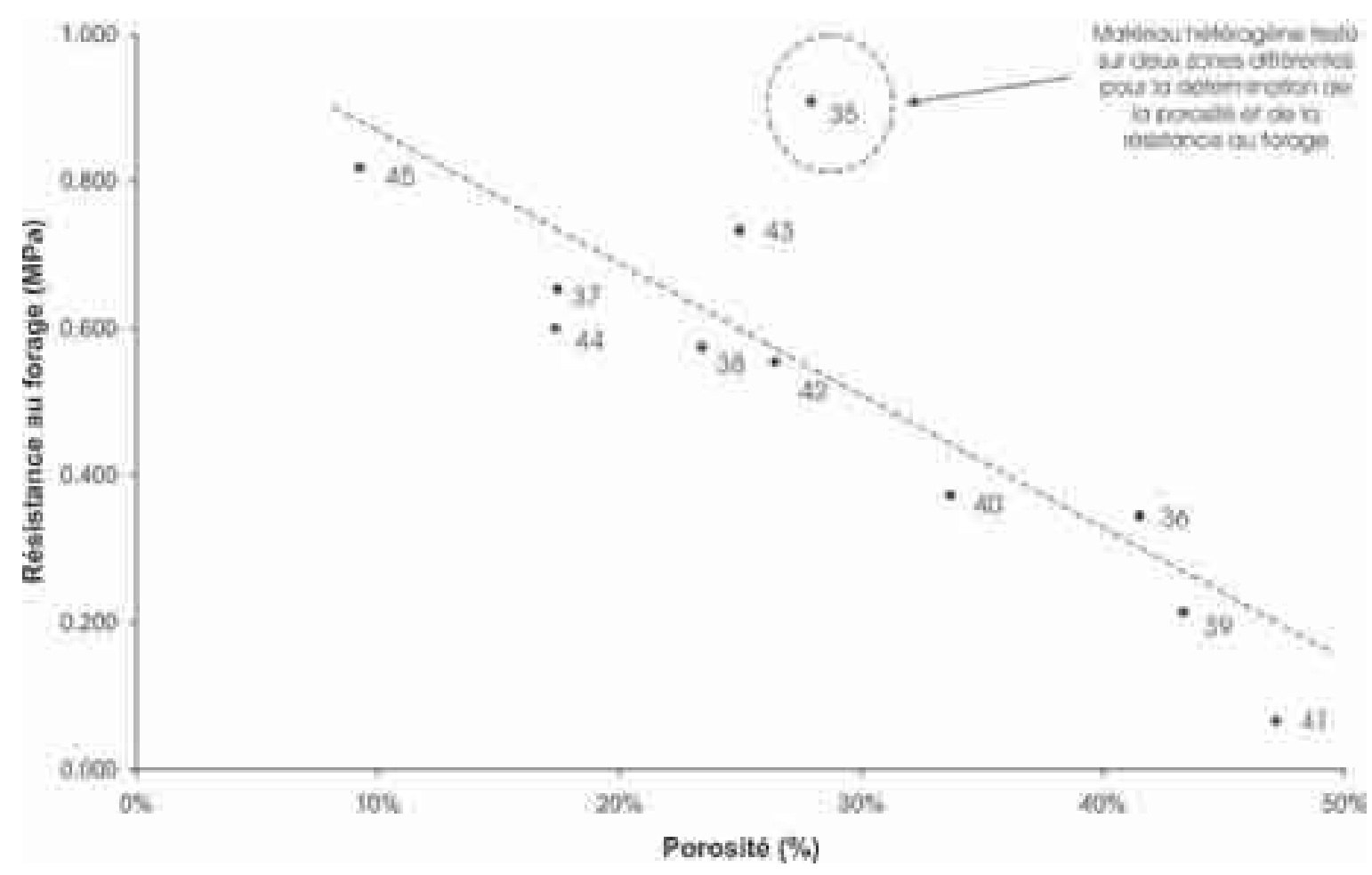

Figure 12 : Exemple d'une tendance observée entre la résistance au forage et la porosité déterminées sur les échantillons de mortiers historiques prélevés sur la Tour Henri VIII à Tournai. L'écart que présente l'échantillon 35 par rapport à la tendance s'explique par le fait que deux morceaux différents ont été testés pour obtenir les deux propriétés.

Figure 12: Example of a tendency observed between drilling strength and porosity determined on the historical mortars sampled on the Henri VIII Tower. The deviation of sample number 35 in comparison to the trend may be explained by the fact that two different pieces of the same sample were tested to obtain both properties.

de contribuer à la formulation des mortiers contemporains à employer dans leur restauration. Cette méthodologie devrait permettre de mieux comprendre le comportement à court, moyen et long termes des mortiers afin d'améliorer les interventions contemporaines, et ce notamment en termes de compatibilité avec l'environnement antique.

\section{Étude des tuiles de l'église Saint-Eleuthère d'Esquelmes}

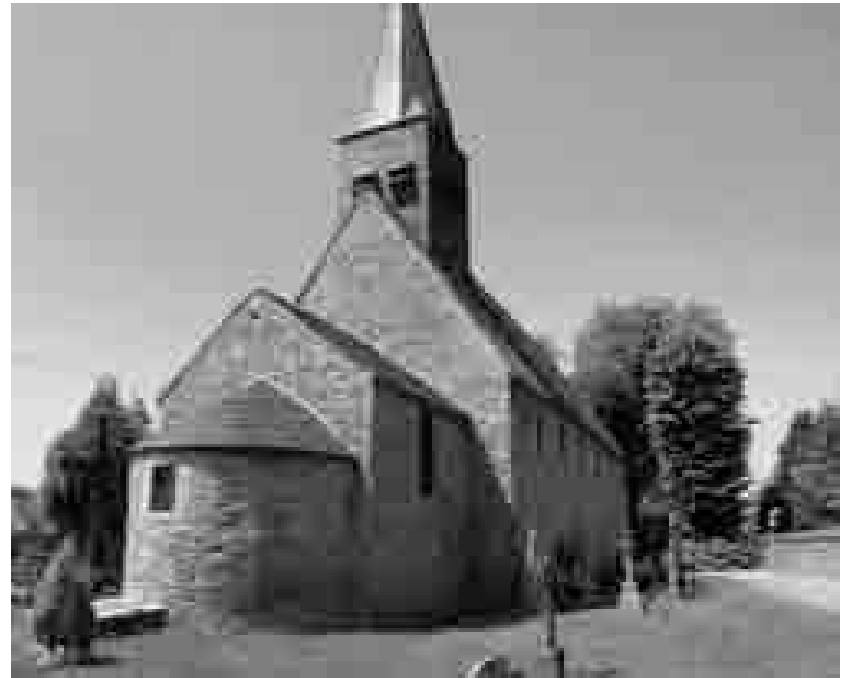

L'Église Saint-Eleuthère d'Esquelmes en Belgique (fig. 13), de style roman, date du viII ${ }^{\mathrm{e}}$ siècle et serait peut-être, la plus ancienne église de Belgique, voir d'Europe Occidentale [Delecluse, 1990].

Le Service de Génie Civil et Mécanique des Structures de l'Université de Mons a été sollicité dans le cadre de la restauration de cet édifice pour évaluer rapidement la qualité de tuiles anciennes afin de déterminer si l'ensemble des tuiles constitutives de la toiture actuelle nécessitait d'être remplacé ou pouvait être conservé. Une importante décision

Figure 13: (Voir planche couleur) Église Saint-Eleuthère à Esquelmes, Belgique.

Figure13: (See colour plate) Saint-Eleuthère Church in Esquelmes, Belgium. 
Figure 14: (Voir planche couleur) Illustration des trois tuiles historiques en provenance de l'église d'Esquelmes. (Dimensions approximatives 330 x 170 x $18 \mathrm{~mm})$.

Figure 14: (See colour plate) Illustration of three historical tiles coming from the church of Esquelmes. (Approximate Dimensions $330 \times 170 \times 18 \mathrm{~mm}$ ).

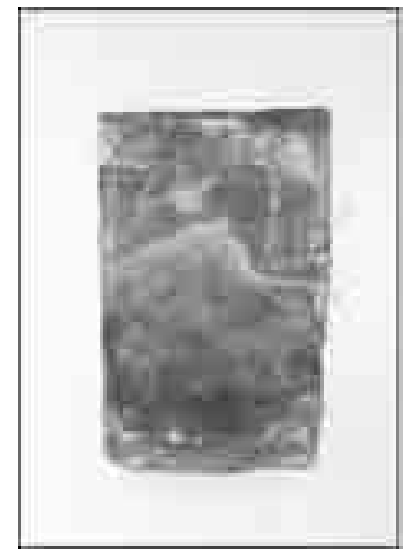

devait donc être prise en ce qui concerne ces tuiles dont l'origine remonterait au moyen-âge. Le propriétaire souhaitait le remplacement total de la couverture par des tuiles contemporaines. Les architectes en charge du chantier proposaient la conservation de la couverture et uniquement le remplacement des éléments dégradés. Une étude comparative de plusieurs tuiles historiques avec divers matériaux contemporains a été menée afin d'apporter des critères scientifiques pouvant justifier l'une ou l'autre solution. Il est important de signaler qu'aucune méthode de tests normalisée n’auraient permis de caractériser les tuiles historiques. L'ensemble de la méthodologie a ainsi été mise en application sur trois tuiles historiques (fig . 14) et sur six tuiles contemporaines diverses ayant déjà été mises en œuvre dans d'autres chantiers de restauration dont celui de la Cathédrale de Tournai. Les principaux résultats des essais de caractérisation sont repris dans le tableau 2.

L'analyse des résultats des essais ne montre pas de dispersion réellement significative des diverses propriétés en fonction des matériaux étudiés. Les tuiles historiques présentent des valeurs de densité légèrement plus faibles, et des porosités un peu plus élevées que les tuiles contemporaines. Par contre les valeurs de résistance sont tout à fait équivalentes. Les vitesses soniques apparaissent quant à elles nettement plus élevées sur les tuiles historiques que sur les tuiles qui ont été sélectionnées pour les travaux de restauration de la Cathédrale de Tournai. Dans le cas précis de l'application proposée, la méthodologie a permis de répondre très rapidement - c'est-à-dire en moins de 48 heures ! - à une demande précise de la Région Wallonne, à savoir les tuiles de l'Eglise Saint-Eleuthère d'Esquelmes présentent-elles des propriétés physico-mécaniques qui nécessitent leur remplacement par des matériaux contemporains? Les propriétés déterminées sur ces matériaux et comparées à celles d'autres matériaux de substitution n'ont pas mis en évidence de valeurs justifiant le remplacement absolu des tuiles de la couverture. L'issue de l'étude a donc été favorable aux
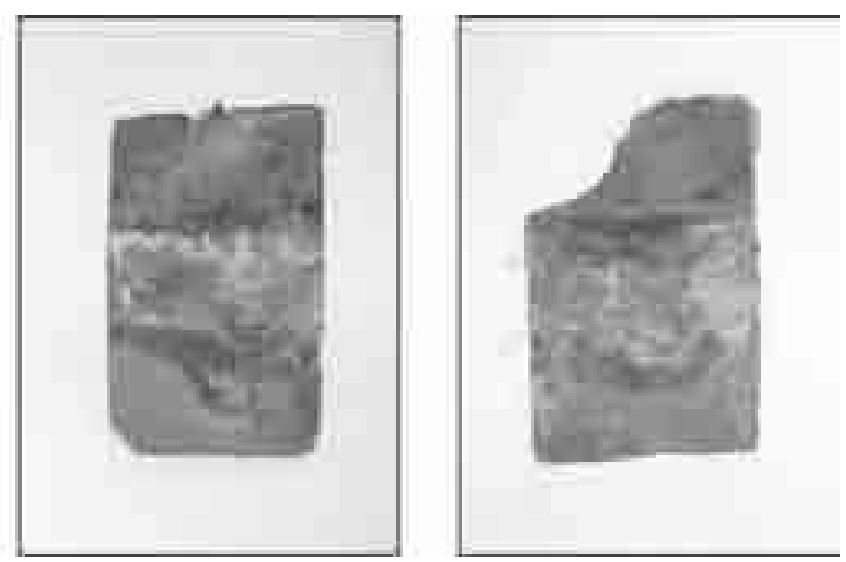

tuiles historiques qui ont pu être sauvegardées aux dépens des tuiles contemporaines. Cette étude a donc permis à la Région Wallonne de prendre les dispositions nécessaires pour conserver ce patrimoine historique.

Notons encore que l'ensemble des propriétés déterminées à l'aide de la méthodologie décrite permet de mettre en évidence des tendances particulières marquées notamment entre la densité et la porosité en fonction de la densité absolue (fig. 15), mais aussi de trier les divers matériaux sur base de divers critères, et même de les classer par familles (fig. 16). La réalisation d'une telle étude sur une plus large gamme de tuiles, d'origines et d'époques diverses, de divers types de processus de fabrication permettrait certainement d'apporter d'autres informations utiles aux scientifiques de tous horizons amenés à étudier ce type de matériaux. Cette approche est également envisagée sur tout autre type de matériaux en terre cuite, tels que les briques et s'intégrerait parfaitement à d'autres études archéologiques telles que celle présentée dans les travaux de thèse de doctorat de Sosnowska (2011).

\section{Étude des matériaux de l'église de Racour}

L'église Saint-Christophe de Racour, en Belgique, (fig. 17) est un édifice romano-gothique édifié en moellons de Quartzite d'Overlaar, en Pierre de Gobertange et en Tuffeau de Lincent. Cette église est caractérisée par une importante tour-porched u XIV $v^{\mathrm{e}}$ siècle flanquée d'une tourelle d'escalier et élevée sur trois niveaux rétrécissant vers le haut. La nef de trois travées, le transept et le chœur ont été construits au $X^{e}{ }^{e}$ siècle. Le chœur et l'abside ont été enduits et ornés de pilastres en stucs à motifs rocailles au XVIII ${ }^{e}$ siècle et sont couverts par un plafond qui se prolonge sur la croisée du transept et la nef. La charpente, enduite, reste visible dans les bas-côtés. Le mobilier comprend notamment des fonts 


\begin{tabular}{|c|c|c|c|c|c|c|c|c|c|c|c|c|}
\hline Réf. & Marque & \multicolumn{2}{|c|}{$\begin{array}{c}\text { Origine des tuiles ou mise en } \\
\text { application }\end{array}$} & d & $\phi(\%)$ & $\begin{array}{c}\mathrm{C}(\mathrm{gr} / \\
\left.\mathrm{m}^{2} / \mathrm{s} 1 / 2\right)\end{array}$ & $\mathrm{Vp}(\mathrm{m} / \mathrm{s})$ & $\mathrm{HL}$ & $\varepsilon(\mathrm{MPa})$ & $\varphi\left({ }^{\circ}\right)$ & $\begin{array}{l}\mathrm{C} 1 \\
(\mathrm{~N}) \\
\end{array}$ & $\begin{array}{c}\mathrm{Dr} \\
(\mathrm{MPa}) \\
\end{array}$ \\
\hline 1 & Tuilehi storique & \multirow{3}{*}{\multicolumn{2}{|c|}{ égliseS t-Eleuthèred 'Esquelmes }} & 1.796 & 31.6 & 121 & 2333 & 507 & 38.8 & 32.3 & 655 & 0.458 \\
\hline 2 & Tuilehi storique & & & 1.927 & 26.9 & 53 & 2667 & 559 & 58.1 & 32.7 & 1431 & 0.682 \\
\hline 3 & Tuilehi storique & & & 1.817 & 30.5 & 74 & 2539 & 514 & 41.7 & 31.2 & 923 & 0.394 \\
\hline 4 & Marcke & \multirow{6}{*}{$\begin{array}{l}\text { Cathédrale } \\
\text { de Tournai }\end{array}$} & \multirow{2}{*}{$\begin{array}{l}\text { Ancienner estaura- } \\
\text { tion années } 70-80\end{array}$} & 1.863 & 27.1 & 35 & 933 & 568 & 20.3 & 51.3 & 929 & 0.577 \\
\hline 5 & Marcke & & & 1.893 & 26.1 & 38 & 829 & 546 & 20.4 & 39.7 & 572 & 0.600 \\
\hline 6 & Blache & & $\begin{array}{l}\text { ChapelleS t-Louis } \\
2010\end{array}$ & 1.989 & 25.3 & 147 & 1226 & 602 & 26.9 & 76.0 & 1322 & 1.187 \\
\hline 7 & Pommardt erreal & & \multirow{3}{*}{$\begin{array}{l}\text { Envisagéesp ourla } \\
\text { restauration de la } \\
\text { Chapelle St Louis } \\
\text { mais non retenues }\end{array}$} & 2.101 & 20.1 & 54 & 2128 & 644 & 25.0 & 49.3 & 1256 & 1.267 \\
\hline 8 & Pommardt erreal & & & 2.041 & 22.3 & 59 & 2450 & 619 & 26.7 & 53.8 & 1453 & 1.411 \\
\hline 9 & Pommardt erreal & & & 2.093 & 21.2 & 56 & 2338 & 588 & 27.3 & 51.3 & 1339 & 1.540 \\
\hline
\end{tabular}

Tableau 2 : Propriétés déterminées sur les différentes tuiles en terre cuite étudiées. $d$ : densité $-\phi$ : Porosité - $C$ : Coefficient de capillarité $-V \mathrm{p}:$ Vitesse sonique $-\mathrm{HL}$ : Dureté Leeb $-\varepsilon$ : énergie spécifique intrinsèque $-\varphi$ : angle de frottement interne $-\mathrm{C}_{1}:$ Coefficient de pente du couple en fonction de l'avance en micro-forage $-\mathrm{D}_{\mathrm{r}}$ : Résistance au forage.

Table 2: Properties determined on the various studied terra cotta tiles.

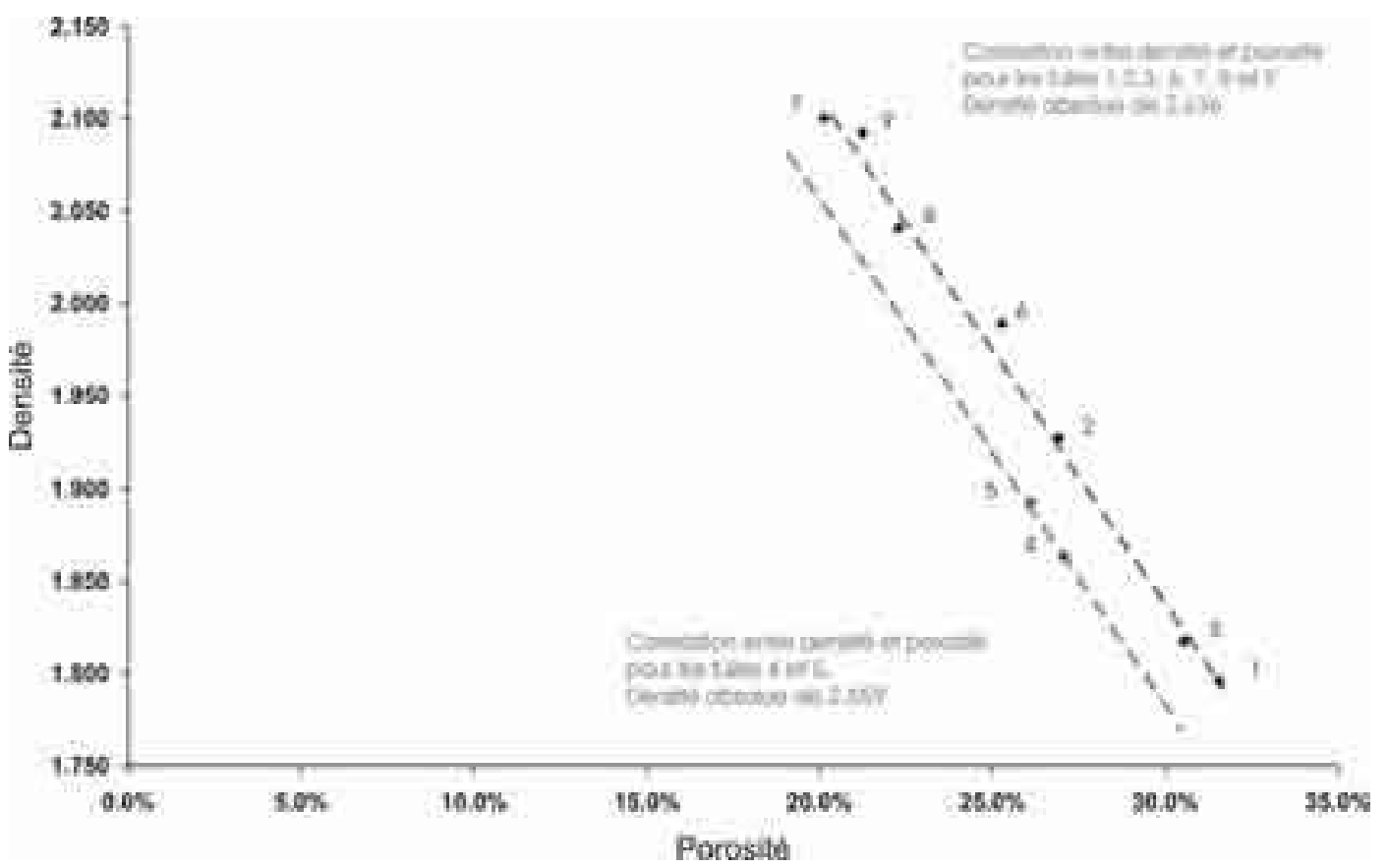

Figure 15 : (Voir planche couleur) Exemple de corrélations entre la densité et la porosité de tuiles en terre cuite qui apparaissent influencées par la densité absolue des matériaux constitutifs. Figure 15: (See colour plate) Example of correlations between the density and the porosity of terra cotta tiles which appear influenced by the absolute density of constitutive materials.

baptismaux gothiques du $\mathrm{Xv}^{\mathrm{e}}$ siècle et un bénitier en calcaire orné de feuilles d'eau daté du XvI si ècle.

La restauration de l'église Saint-Christophe a débuté, il y une vingtaine d'années, par la réfection de la tour et des toitures. Depuis lors, les murs en tuffeau de Lincent se sont fortement dégradés à cause des intempéries, et les contreforts du chœur présentent de nombreuses fissures qui risquent de nuire à l'intégrité du bâtiment. La phase de restauration des parements en Tuffeau de Lincent est restée en souffrance durant de nombreuses années.

Le Tuffeau de Lincent était à l'origine exploité dans les carrières locales qui ont aujourd'hui disparu pour diverses raisons. Si de nombreuses restaurations ont pu être réalisées par le passé soit par retournement des pierres de parement, soit grâce au réemploi de matériaux prélevés sur d'anciens bâtiments en ruine, ou encore via la réouverture temporaire d'un ancien site d'extraction, les travaux actuels nécessitent l'utilisation de matériaux de substitution. Les critères généralement considérés pour le choix des pierres de remplacement ne se basent pas du tout sur une approche scientifique, mais bien sur des critères esthétiques (essentiellement la couleur), et surtout sur des aspects économiques [Dresen et Dusar, 2004]. Depuis le xix ${ }^{\mathrm{e}}$ siècle, la majeure partie des bâtiments classés en Région Wallonne ou en Belgique et nécessitant le remplacement de pierres calcaires claires locales, sont restaurés avec des pierres de France dont les plus courantes, la Pierre de la Savonnière, la Pierre de Massangis 
Figure 16 : Exemple de représentation graphique permettant la classification des tuiles testées.

Figure 16: Example of a chart allowing the classification of the tested tiles.

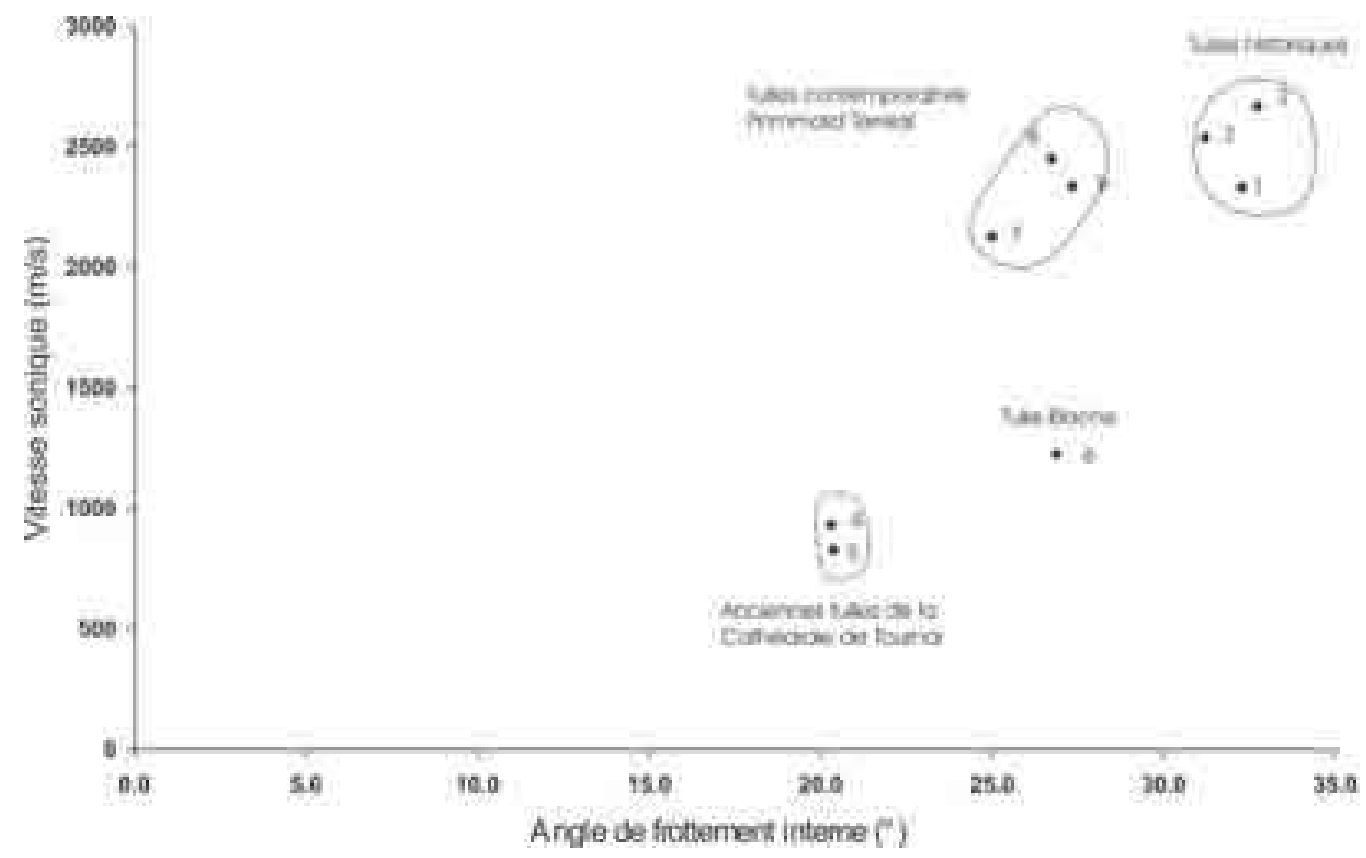

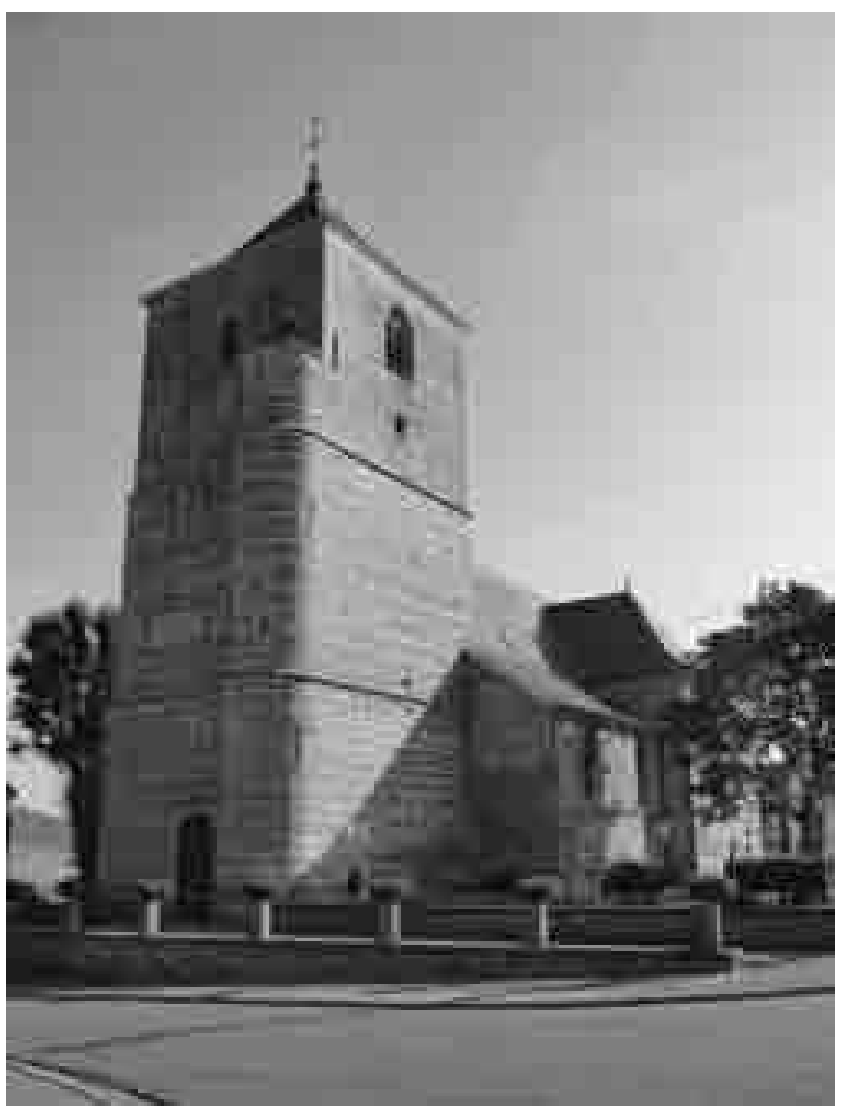

Figure 17 : (Voir planche couleur) L'église Saint-Christophe de Racour, Belgique.

Figure 17: (See colour plate) Saint-Christophe Church in Racour, Belgium. ou la Pierre d'Euville, sont exploitées dans d'imposantes carrières du nord de la Bourgogne.

Tout chantier de restauration nécessiterait pourtant une étude préalable pour choisir les matériaux de substitution sur base de critères plus scientifiques. Certains proposent d'effectuer l'analyse pétrographique des matériaux altérés et des matériaux potentiels de substitution avant toute restauration de manière à sélectionner ceux qui sont les plus fidèles aux matériaux d'origine tant géologiquement que minéralogiquement [Dresen et Dusar, 2004]. Nous prônons à l'université de Mons, outre le respect esthétique (notamment la couleur et la structure macroscopique), que les matériaux de substitution devraient en plus être choisis de manière à garantir la compatibilité des matériaux d'un point de vue physico-mécanique et assurer un vieillissement adéquat des matériaux.

Le cahier des charges du chantier de restauration de cette église prévoit notamment le remplacement du Tuffeau de Lincent altéré soit par du Tuffeau de Maastricht, soit par de la Pierre de la Savonnière. La méthodologie de caractérisation décrite ci-dessus a été mise en application pour étudier et comparer les deux matériaux de substitution proposés au matériau d'origine (tableau 3). L'étude comparative de ces trois matériaux a permis de mettre en évidence que le Tuffeau de Maastricht n'est pas adapté pour la restauration de cet édifice même si des cas de restauration avec ce matériau existe sur d'autres bâtiments patrimoniaux de la région. Le Tuffeau de Maastricht présente notamment une résistance mécanique trop faible et un coefficient de capilla- 
rité beaucoup trop élevé. De plus, il présente une teinte qui le distingue nettement du Tuffeau de Lincent. La mise en application de ce type de pierre sur des édifices en Tuffeau de Lincent a permis de constater que les deux pierres ne vieillissent pas de manière similaire. Le tuffeau de Maastricht se couvre assez rapidement d'un calcin de couleur grisâtre qui a pour conséquence l'apparition d'un rapiéçage inesthétique dans certains cas (fig. 18).

En ce qui concerne la Pierre de la Savonnière, même si l'étude réalisée a permis de vérifier que sa résistance mécanique est du même ordre de grandeur que celle du matériau d'origine et que la porosité et la capillarité sont plus faibles, la comparaison de sa structure macroscopique et de sa couleur avec celles du tuffeau de Lincent laisse présager un vieillissement différent des deux matériaux. La Pierre de la Savonnière un peu plus claire et présentant des pores plus grossiers risque fort de se patiner et se ternir suite à la pollution atmosphérique contrairement au tuffeau de Lincent, qui vu son comportement gélif, a tendance à conserver sa couleur naturelle par perte progressive de la patine superficielle.

Le parcours de la base de données du Service de Génie Civil et Mécanique des Structures et de sa lithothèque a permis de trouver d'autres matériaux de substitution que les deux initialement prévus dans le cahier des charges du chantier de restauration et qui présentent non seulement des caractéristiques mécaniques compatibles avec le tuffeau de Lincent, mais qui en plus sont esthétiquement plus proches que ceux proposés dans le cahier des charges (Dagrain, 2011). Parmi les matériaux de la base de données citons les trois pierres de France suivantes : la pierre de Richemont Jaune, la pierre de Tervoux, et la pierre de Chauvigny. Les caractéristiques physico-mécaniques de ces trois matériaux sont également reprises dans le tableau 3. La figure 19 permet de visualiser les aspects esthétiques des différentes pierres mentionnées.

Remarquons encore que dans tous les cas, la densité des matériaux de substitution potentiels étant nettement plus élevée (d'un peu plus de $55 \%$ pour la Pierre de la Savonnière à près de $80 \%$ dans le cas de la Pierre de Chauvigny), l'utilisation de matériaux de substitution plus denses nécessiterait très certainement de vérifier la portance des fondations et s'assurer que l'augmentation de la charge des parements suite à leur restauration ne risquent pas d'engendrer d'autres dégâts structurels au niveau de l'ouvrage.

\section{Conclusion}

La méthodologie de caractérisation physico-mécanique sur petits échantillons prélevés sur les bâtiments patrimo-
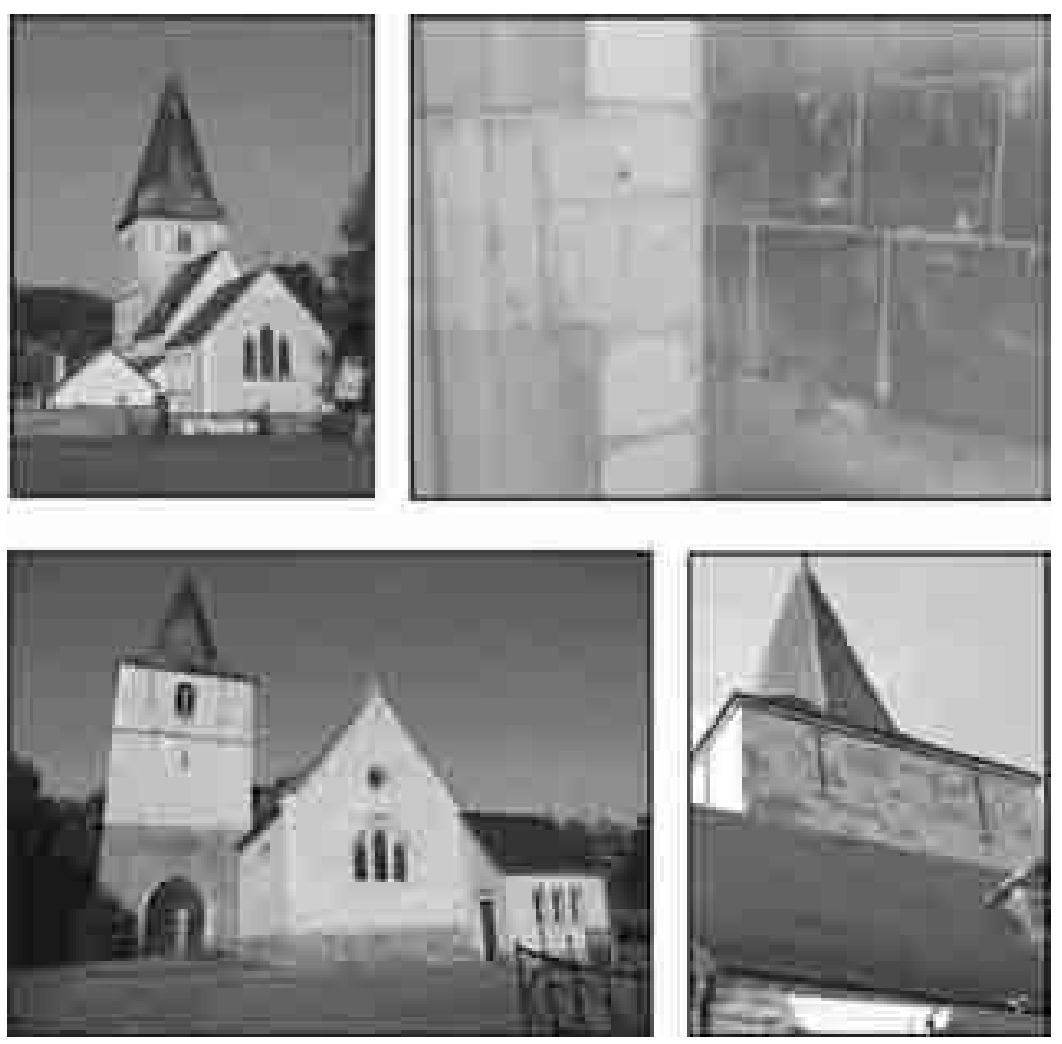

Figure 18: (Voir planche couleur) Deux exemples de mise en ouvre de Tuffeau de Maastricht en remplacement de Tuffeau de Lincent. En haut, l'Eglise de Wezeren où le clocher a été entièrement restauré en Tuffeau de Maastricht, tandis que le cour et la nef ont été conservé en Tuffeau de Lincent. La jonction entre les deux parties du bâtiment montre clairement la différence de patine entre les deux types de tuffeau. En bas, l'Eglise de Walsbets montre un rapiéçage très marqué suite au remplacement ponctuel de bloc de Tuffeau de Lincent par du Tuffeau de Maastricht.

Figure 18: (See colour plate) Two examples of substitution of Lincent Limestone by Maastricht Limestone. In top, the Church of Wezeren (Belgium) where the bell-tower was entirely restored out with Maastricht Limestone, while the heart and the nave were preserved in the original Lincent Limestone The junction both parts of the building clearly shows the difference of patina between the both types of limestone. In bottom, the Church of Walsbets (Belgium) shows a patchwork very marked due to the substitution of the original Lincent Limestone by Maastricht Limestone. 
Figure 19 : (Voir planche couleur) Illustration des différents matériaux dont les propriétés sont comparées dans le tableau 3. Figure 19: (See colour plate) Illustration of the various materials whose properties are compared in table 3 .

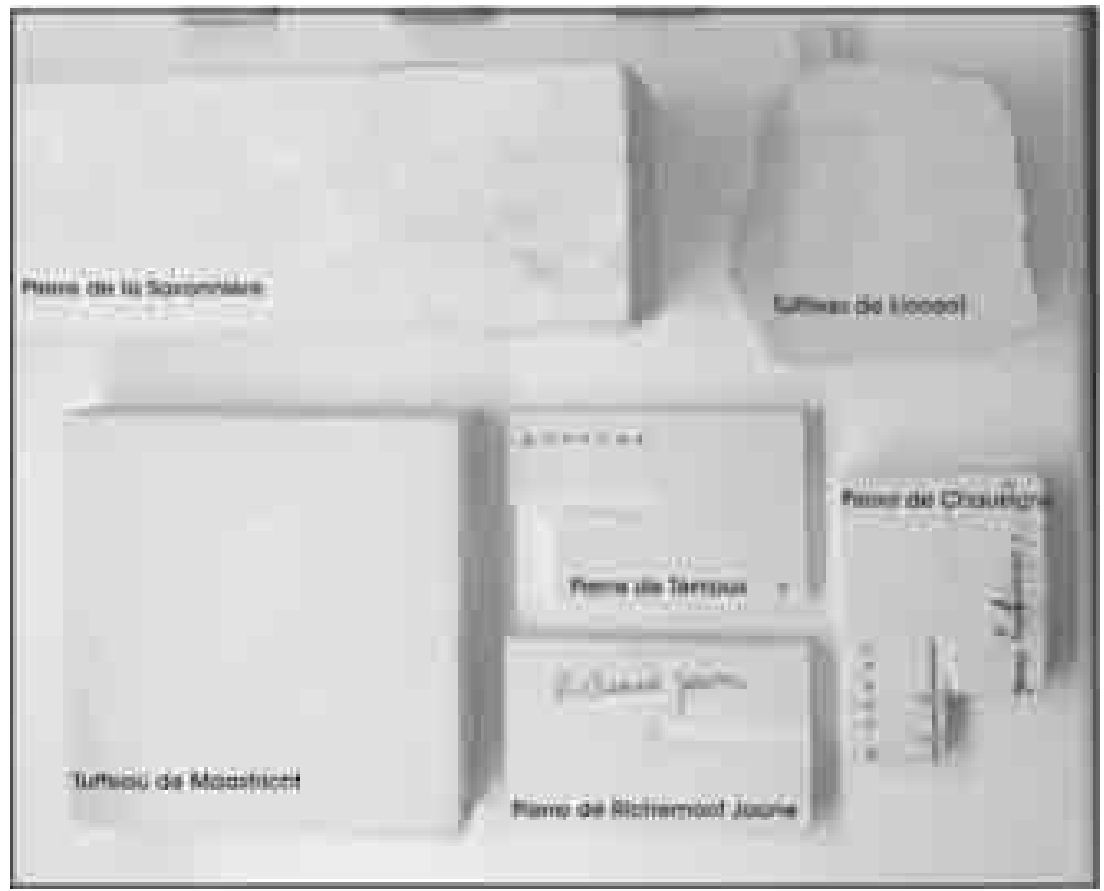

\begin{tabular}{|c|c|c|c|c|c|c|c|c|c|}
\hline & Réf. & $\mathrm{d}$ & $\phi(\%)$ & $\begin{array}{c}\mathrm{C}\left(\mathrm{gr} / \mathrm{m}^{2} /\right. \\
\mathrm{s} 1 / 2)\end{array}$ & $\mathrm{Vp}(\mathrm{m} / \mathrm{s})$ & $\mathrm{HL}$ & $\varepsilon(\mathrm{MPa})$ & $\mathrm{C} 1(\mathrm{~N})$ & $\begin{array}{c}\mathrm{Dr} \\
(\mathrm{MPa})\end{array}$ \\
\hline Tuffeau de Lincent & & 1.180 & 55 & 143 & 1481 & 322 & 15.1 & 138 & 0.293 \\
\hline Pierres du cahier des & Tuffeaud eM aastricht & 1.313 & 52.4 & 3570 & 1868 & 204 & 2.2 & 17 & 0.008 \\
\hline & Pierred ela $S$ avonnière & 1.850 & 32.1 & 103 & 3389 & 310 & 18.3 & 224 & 0.250 \\
\hline Autresp ierresd e & Pierred eR ichemont & 1.897 & 30.1 & - & 2416 & 280 & 13.2 & - & 0.083 \\
\hline remplacement & Pierred e Tervoux & 2.084 & 22.7 & - & 2843 & 384 & 15.5 & - & 0.157 \\
\hline potentielles & Pierred eCha uvigny & 2.103 & 22.2 & - & 3224 & 415 & 23.7 & - & 0.459 \\
\hline
\end{tabular}

Tableau 3 : Propriétés déterminées sur diverses pierres dans le cadre de la restauration de l'église de Racour. $d:$ densité $-\phi:$ Porosité $C$ : Coefficient de capillarité $-V \mathrm{p}$ : Vitesse sonique - Edyn : Module de Young Dynamique - HL : Dureté Leeb $-\varepsilon$ : énergie spécifique intrinsèque $-\mathrm{C}_{1}$ :Coeffi cient de pente du couple en fonction de l'avance en micro-forage $-\mathrm{D}_{\mathrm{r}}$ : Résistance au forage.

Table 3: Properties determined on various stones within the framework of the restoration of the church of Racour.

niaux ou sur des sites de fouilles archéologiques permet d'accéder à plusieurs propriétés physiques et mécaniques. Les diverses applications qui ont déjà pu être réalisées jusqu'à présent illustrent les potentialités de la méthode non seulement à fournir aux archéologues des informations utiles sur les matériaux rencontrés dans leurs études, mais aussi à aider les architectes et entrepreneurs à choisir les matériaux compatibles avec le patrimoine historique bâti, ou encore aux ingénieurs de prévoir l'effet d'une modification partielle ou totale de la répartition des charges sur la structure globale d'un monument à restaurer. La méthodologie commence à se faire connaître pour les nombreux avantages qu'elle présente comparativement aux méthodes classiques de caractérisation. Parmi ces avantages, insistons sur le fait que la caractérisation physico-mécanique (i) se base sur des échantillons de taille réduite ne devant pas forcément présenter de taille ou forme prédéfinie, (ii) qu'elle est relativement 
rapide, (iii) qu'elle peut fournir l'ensemble des propriétés en réalisant tous les essais sur le même échantillon.

\section{Bibliographie}

Beck, K., Al-Mukhtar, M. et Rozenbaum, O., 2003 - Pierres des monuments historiques : caractérisations et mécanismes d'altération du tuffeau. Environnement et Patrimoine: Les nouvelles donnes. Association Française de Génie Civil.

Carré, A., Mzabi, S. et Woehl, P., 2005 - Caractérisation d'un milieu poreux par mouillabilité. In Proc. 13 Journée d'Etude sur l'adhésion JADH 2005, 25-30 septembre, Belwiller, France.

Dagrain, F., Richard, T. et Germay, C., 2006 - The Rock Strength Device : A scratching apparatus to determine rock properties. In Proc. The 7th National Congress on theoretical and applied Mechanics NCTAM 2006, May 29-30, Faculté Polytechnique de Mons, Mons, Belgium.

Dagrain, F. et Richard, T., 2006 - On the influence of PDC wear and rock type on cutting efficiency. Eurock 2006, May 9-12, Liège, Belgium.

DAgraIn, F., 2007 - Le test de grattage, une technique alternative pour la détermination rapide de la résistance en compression des géomatériaux. In Proc. Symposium " de la bonne idée à la réalisation pratique... ». 6 Journée de l'Innovation du CSTC. Le 28 novembre, Ecole Royale Militaire, Bruxelles, Belgique.

Dagrain, F., Demelenne, M. et Scaillet, J.-C., 2010a - Widescale program on the suitability of mortar formulations for interventions on heritage buildings in the Walloon region. In Proc. 8th International Masonry Conference, July, Dresden, Germany.

Dagrain, F. et Descamps, T., 2010b - Less-destructive testing of masonry materials : a comparison between scratching and drilling approaches. In Proc. 8th International Masonry Conference, July..., Dresden, Germany.

Dagrain, F., 2011 - Nouvelles techniques de caractérisation de la pierre naturelle dans le cadre de travaux de restauration, Pierre Actual n ${ }^{\circ} 897$, septembre, p. 76-85.

Delecluse, E., 1990 - Esquelmes sur Escaut et son église millénaire.

Del Monte, E. et Vignoli, A., 2008 - In situ mechanicl characterization of the mortar in masonry buildings with DRMS, dans Proc. Interantional RILEM Conference on Site Assessment of Concret, Masonry and Timber Structures SACoMaTis 2008, 1-2 september, Varenna, Italy.
Demelenne, M., 2010 - Caractéristiques mécaniques des mortiers de la Tour Henry VIII à Tournai. Non interne publié. Février.

Demelenne, M., 2011 - La caractérisation physico-mécanique des mortiers historiques : une méthodologie nouvelle au service de l'archéologie ?, dans Proc. XVIII ${ }^{\mathrm{e}}$ Colloque d'archéométrie du GMPCA, 11-15 avril, Liège, Belgique.

Deramaix, I. et Dosogne, M., 2009 - Tournai : interventions archéologiques à la tour Henri VIII. Nouvelles de la Société royale d'histoire et d'archéologie de Tournai, mai-juin, 8/3, p. 28-34.

Dosogne, M., 2009 - Tournai : intervention préventive sur le site de la tour Henry VIII, Chronique de l'archéologie wallonne, 16, p. 65-67.

Dresen, R. et Dusar, M., 2004 - Historical building stones in the province of Limburg (NE Belgium): role of petrography in provenance and durability assessment. Materials Characterization, $\mathrm{n}^{\circ} 53$, p. 273-287.

Fernandes, F. et Lourenço, P.B., 2007 - Evaluation of the compressive strength of Ancient clay bricks using microdrilling. Journal of materials in civil engineering (C) ASCE, September, pp. 791-800.

Fratini, F., Rescic, S. et Tiano, P. 2006 - A new portable system for determining the state of conservation of monumental stones, Materials and Structures, 39, p. 139-147.

LeEb, D., 1986 - Definition of the hardness value "L". VDI Berichte 583, p. 109-133.

Pamplona, M., Kocher, M., Snethlage, R., Aires Barros L., 2007 - Drilling resistance : overview and outlook, Z. dt. Ges. Geowiss., 158/3, p. 665-676.

Rummel, F. et van Heerden, W.L., 1978 - Suggested Methods for determining sound velocity. International Journal of Rock Mechanics and Mining Sciences \& Geomechanics Abstracts, Vol. $15, \mathrm{n}^{\circ} 2$, p. 53-58.

Sosnowska, Ph., 2011 - Contribution à l'histoire de l'architecture à Bruxelles : étude archéologique, technique et historique des matériaux de construction utilisés dans le bâti bruxellois (XIII ${ }^{e}$-XIX siècles). In Proc. XVIII ${ }^{\mathrm{e}}$ Colloque d'archéométrie du GMPCA, 11-15 avril, Liège, Belgique.

Verwaal et Mulder, 1993 - Estimating rock strength with the Equotip hardness tester. International Journal of Rock Mechanics and Mining Sciences, Vol. 30, p. 659-662.

Webb, P.A., 2001 - Volume and Density Determinations for Particle Technologists. Micromeritics Instrument Corporation. February. 